\title{
NONEQUILIBRIUM DYNAMICS OF INTERFACES AND LINES
}

\author{
Mehran Kardar \\ Department of Physics \\ Massachusetts Institute of Technology \\ Cambridge, Massachusetts 02139, USA
}

These notes are prepared for a set of lectures delivered at the The 4th CTP Workshop on Statistical Physics: "Dynamics of Fluctuating Interfaces and Related Phenomena", at Seoul National University, Korea. The lectures examine several problems related to non-equilibrium fluctuations of interfaces and flux lines. The first two introduce the phenomenology of depinning, with particular emphasis on interfaces and contact lines. The role of the anisotropy of the medium in producing different universality classes is elucidated. The last two lectures focus on the dynamics of lines, where transverse fluctuations are also important. We shall demonstrate how various non-linearities appear in the dynamics of driven flux lines. The universality classes of depinning, and also dynamic roughening, are illustrated in the contexts of moving flux lines, advancing crack fronts, and drifting polymers.

\section{Depinning of Interfaces}

\subsection{Introduction and Phenomenology}

Depinning is a non-equilibrium critical phenomenon involving an external force and a pinning potential. When the force is weak the system is stationary, trapped in a metastable state. Beyond a threshold force the (last) metastable state disappears and the system starts to move. A simple example is provided by a point mass on a rough table. The mass is stationary until the external force $F$ exceeds that of static friction $F_{c}$. Larger forces lead to an initial period of acceleration, before the motion settles to a uniform velocity due to viscous forces. In the latter is proportional to velocity, the ultimate velocity of the point close to threshold behaves as $v \propto\left(F-F_{c}\right)$.

While there are many other macroscopic mechanical examples, our main interest comes from condensed matter systems such as Charge Density Waves $(\mathrm{CDWs})^{1}$, interfaces ${ }^{2}$, and contact lines ${ }^{3}$. In CDWs, the control parameter is the external voltage; a finite CDW current appears only beyond a threshold applied voltage. Interfaces in porous media, domain walls in random magnets, are stationary unless the applied force (magnetic field) is sufficiently strong. A key feature of these examples is that they involve the collective depinning of many degrees of freedom that are elastically coupled. As such, these problems belong to the realm of collective critical phenomena, characterized by universal 


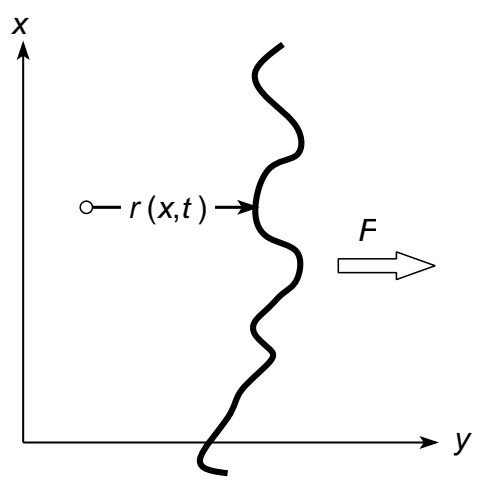

Figure 1. Geometry of the line in two dimensions.

scaling laws. We shall introduce these laws and the corresponding exponents below for the depinning of a line (interface or contact line).

Consider a line in two dimensions, oriented along the $x$ direction, and fluctuating along a perpendicular direction $r$. The configuration of the line at time $t$ is described by the function $r(x, t)$. The function $r$ is assumed to be single valued, thus excluding configurations with overhangs. In many cases ${ }^{2}$, where viscous forces dominate over inertia, the local velocity of a point on the curve is given by

$$
\frac{d r(x, t)}{d t}=F+f(x, r)+\mathcal{K}[r] .
$$

The first term on the right hand side is a uniform applied force which is also the external control parameter. Fluctuations in the force due to randomness and impurities are represented by the second term. With the assumption that the medium is on average translationally invariant, the average of $f$ can be set to zero. The final term in eq.(1)describes the elastic forces between different parts of the line. Short range interactions can be described by a gradient expansion; for example, a line tension leads to $\mathcal{K}[r(x)]=\nabla^{2} r$ or $\mathcal{K}[r(q)]=-q^{2} r(q)$ for the Fourier modes. The surface of a drop of non-wetting liquid terminates at a contact line on a solid substrate ${ }^{3}$. Deformations of the contact line are accompanied by distortions of the liquid/gas surface. As shown by Joanny and de Gennes ${ }^{4}$, the resulting energy and forces are non-local, described by $\mathcal{K}[r(q)]=-|q| r(q)$.

For the case of a surface in three dimensions deformations are described by $r\left(x_{1}, x_{2}\right)$. More generally, we shall consider $r(\mathbf{x})$, where $\mathbf{x}$ is a $d$-dimensional vector. In a similar spirit, we shall generalize the coupling to $\mathcal{K}[r(q)]=$ $-|q|^{\sigma} r(q)$, which interpolates between the above two cases as $\sigma$ changes from one to two. Note, however, that the equation of motion need not originate 
from variations of a Hamiltonian, and may include non-linear couplings which will be discussed later on.

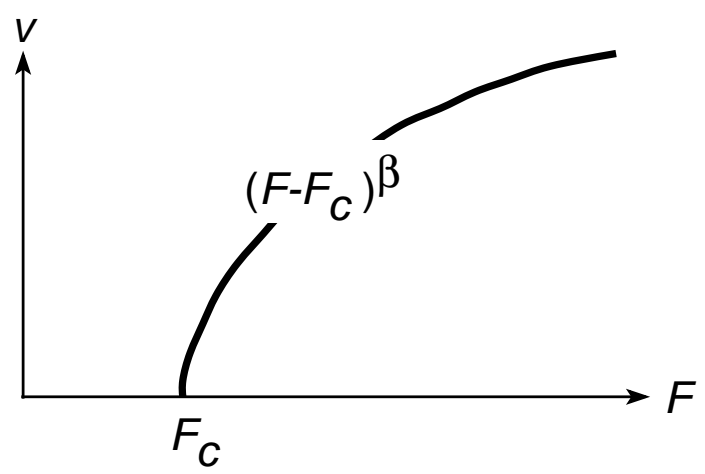

Figure 2. Critical behavior of the velocity.

When $F$ is small, the line is trapped in one of many metastable states in which $\partial r / \partial t=0$ at all points. For $F$ larger than a threshold $F_{c}$, the line is depinned from the last metastable state, and moves with an average velocity $v$. On approaching the threshold from above, the velocity vanishes as

$$
v=A\left(F-F_{c}\right)^{\beta},
$$

where $\beta$ is the velocity exponent, and $A$ is a nonuniversal amplitude. A meanfield estimate for $\beta$ was obtained by Fisher in the context of $\mathrm{CDWs}^{5}$. It corresponds to the limit $\sigma=0$, where every point is coupled to all others, and hence experiences a restoring force proportional to $\langle r(\mathbf{x})\rangle-r(\mathbf{x})$. The resulting equation of motion,

$$
\frac{d r(\mathbf{x})}{d t}=\langle r(\mathbf{x})\rangle-r(\mathbf{x})+F+f(\mathbf{x}, r(\mathbf{x})),
$$

has to be supplemented with the condition $\langle r(\mathbf{x})\rangle=v t$. The self-consistent solution for the velocity indeed vanishes as $\left(F-F_{c}\right)^{\beta}$, with an exponent that depends on the details of the random force. If $f(\mathbf{x}, r(\mathbf{x}))$ varies smoothly with $r$, the exponent is $\beta=3 / 2$, while discontinuous jumps in the force (like a saw-tooth) result in $\beta=1$. In fact the latter is a better starting point for depinning in finite dimensions. This is because of the avalanches in motion (discussed next), which lead to a discontinuous coarse grained force.

The motion just above threshold is not uniform, composed of rapid jumps as large segments of the line depin from strong pinning centers, superposed on 
the slower steady advance. These jumps have a power law distribution in size, cutoff at a correlation length $\xi$ which diverges at the transition as

$$
\xi \sim\left(F-F_{c}\right)^{-\nu} .
$$

The jumps are reminiscent of avalanches in other slowly driven systems. In fact, the depinning can be approached from below $F_{c}$ by monotonically increasing $F$ in small increments, each sufficient to cause a jump to the next metastable state. The size and width of avalanches becomes invariant on approaching $F_{c}$. For example,

$$
\operatorname{Prob}(\text { width of avalanche }>\ell) \approx \frac{1}{\ell^{\kappa}} \hat{\rho}\left(\ell / \xi_{-}\right),
$$

where the cutoff $\xi_{-}$diverges as in Eq.(3). The critical line is a self-affine fractal whose correlations satisfy the dynamic scaling from

$$
\left\langle\left[r(x, t)-r\left(x^{\prime}, t^{\prime}\right)\right]^{2}\right\rangle=\left(x-x^{\prime}\right)^{2 \zeta} g\left(\frac{\left|t-t^{\prime}\right|}{\left|x-x^{\prime}\right|^{z}}\right),
$$

defining the roughness and dynamic exponents, $\zeta$ and $z$ respectively. (Angular brackets reflect averaging over all realizations of the random force $f$.) The scaling function $g$ goes to a constant as its argument approaches $0 ; \zeta$ is the wandering exponent of an instantaneous line profile, and $z$ relates the average lifetime of an avalanche to its size by $\tau(\xi) \sim \xi^{z}$.

Although, the underlying issues of collective depinning for CDWs and interfaces have been around for some time, only recently a systematic perturbative approach to the problem was developed. This functional renormalization group (RG) approach to the dynamical equations of motion was originally developed in the context of CDWs by Narayan and Fisher ${ }^{6}(\mathrm{NF})$, and extended to interfaces by Nattermann et $\mathrm{al}^{7}$. We shall provide a brief outline of this approach starting from Eq.(1). Before embarking on the details of the formalism, it is useful to point out some scaling relations amongst the exponents which follow from underlying symmetries and non-renormalization conditions.

1. As mentioned earlier, the motion of the line close to the threshold is composed of jumps of segments of size $\xi$. Such jumps move the interface forward by $\xi^{\zeta}$ over a time period $\xi^{z}$. Thus the velocity behaves as,

$$
v \sim \frac{\xi^{\zeta}}{\xi^{z}} \sim\left|F-F_{c}\right|^{\nu(z-\zeta)} \quad \Longrightarrow \quad \beta=\nu(z-\zeta) .
$$

2. If the elastic couplings are linear, the response of the line to a static perturbation $\varepsilon(x)$ is obtained simply by considering

$$
r_{\varepsilon}(x, t)=r(x, t)-\mathcal{K}^{-1}[\varepsilon(x)],
$$


where $\mathcal{K}^{-1}$ is the inverse kernel. Since, $r_{\varepsilon}$ satisfies Eq.(1) subject to a force $F+\varepsilon(x)+f\left(x, r_{\varepsilon}\right), r$ satisfies the same equation with a force $F+f(x, r-$ $\left.\mathcal{K}^{-1}[\varepsilon(x)]\right)$. As long as the statistical properties of the stochastic force are not modified by the above change in its argument, $\partial\langle r\rangle / \partial \varepsilon=0$, and

$$
\left\langle\frac{\partial r_{\varepsilon}(x)}{\partial \varepsilon(x)}\right\rangle=-\mathcal{K}^{-1}, \quad \text { or } \quad\left\langle\frac{\partial r_{\varepsilon}(q)}{\partial \varepsilon(q)}\right\rangle=\frac{1}{|q|^{\sigma}} .
$$

Since it controls the macroscopic response of the line, the kernel $\mathcal{K}$ cannot change under RG scaling. From Eqs.(5) and (3), we can read off the scaling of $r(x)$, and the force $\delta F$, which using the above non-renormalization must be related by the exponent relation

$$
\zeta+\frac{1}{\nu}=\sigma
$$

Note that this identity depends on the statistical invariance of noise under the transformation in Eq.(7). It is satisfied as long as the force correlations $\left\langle f(x, r) f\left(x^{\prime}, r^{\prime}\right)\right\rangle$ only depend on $r-r^{\prime}$. The identity does not hold if these correlations also depend on the slope $\partial r / \partial x$.

3. A scaling argument related to the Imry-Ma estimate of the lower critical dimension of the random field Ising model, can be used to estimate the roughness exponent ${ }^{8}$. The elastic force on a segment of length $\xi$ scales as $\xi^{\zeta-\sigma}$. If fluctuations in force are uncorrelated in space, they scale as $\xi^{-(\zeta+1) / 2}$ over the area of an avalanche. Assuming that these two forces must be of the same order to initiate the avalanche leads to

$$
\zeta=\frac{2 \sigma-1}{3} .
$$

This last argument is not as rigorous as the previous two. Nonetheless, all three exponent identities can be established within the RG framework. Thus the only undetermined exponent is the dynamic one, $z$.

\subsection{Functional Renormalization Group}

A field theoretical description of the dynamics of Eq.(1) can be developed using the formalism of Martin, Siggia and Rose ${ }^{9}$ (MSR): Generalizing to a $d$-dimensional interface, an auxiliary field $\hat{r}(\mathbf{x}, t)$ is introduced to implement the equation of motion as a series of $\delta$-functions. Various dynamical response and correlation functions for the field $r(\mathbf{x}, t)$ can then be generated from the functional,

$$
Z=\int \mathcal{D} r(\mathbf{x}, t) \mathcal{D} \hat{r}(\mathbf{x}, t) \mathcal{J}[r] \exp (S)
$$


where

$$
S=i \int d^{d} \mathbf{x} d t \hat{r}(\mathbf{x}, t)\left\{\partial_{t} r-\mathcal{K}[r]-F-f(\mathbf{x}, r(\mathbf{x}, t))\right\} .
$$

The Jacobian $\mathcal{J}[r]$ is introduced to ensure that the $\delta$-functions integrate to unity. It does not generate any new relevant terms and will be ignored henceforth.

The disorder-averaged generating functional $\bar{Z}$ can be evaluated by a saddle-point expansion around a Mean-Field (MF) solution obtained by setting $\mathcal{K}_{M F}[r(\mathbf{x})]=v t-r(\mathbf{x})$. This amounts to replacing interaction forces with Hookean springs connected to the center of mass, which moves with a velocity $v$. The corresponding equation of motion is

$$
\frac{d r_{M F}}{d t}=v t-r_{M F}(t)+f\left[r_{M F}(t)\right]+F_{M F}(v),
$$

where the relationship $F_{M F}(v)$ between the external force $F$ and average velocity $v$ is determined from the consistency condition $\left\langle r_{M F}(t)\right\rangle=v t$. The MF solution depends on the type of irregularity ${ }^{6}$ : For smoothly varying random potentials, $\beta_{M F}=3 / 2$, whereas for cusped random potentials, $\beta_{M F}=1$. Following the treatment of $\mathrm{NF}^{6,10}$, we use the mean field solution for cusped potentials, anticipating jumps with velocity of $O(1)$, in which case $\beta_{M F}=1$. After rescaling and averaging over impurity configurations, we arrive at a generating functional whose low-frequency form is

$$
\begin{aligned}
\bar{Z}=\int & \mathcal{D} R(\mathbf{x}, t) \mathcal{D} \hat{R}(\mathbf{x}, t) \exp (\tilde{S}), \\
\tilde{S}=- & \int d^{d} \mathbf{x} d t\left[F-F_{M F}(v)\right] \hat{R}(\mathbf{x}, t) \\
& -\int \frac{d^{d} \mathbf{q}}{(2 \pi)^{d}} \frac{d \omega}{2 \pi} \hat{R}(-\mathbf{q},-\omega)\left(-i \omega \rho+|\mathbf{q}|^{\sigma}\right) R(\mathbf{q}, \omega) \\
& +\frac{1}{2} \int d^{d} \mathbf{x} d t d t^{\prime} \hat{R}(\mathbf{x}, t) \hat{R}\left(\mathbf{x}, t^{\prime}\right) C\left[v t-v t^{\prime}+R(\mathbf{x}, t)-R\left(\mathbf{x}, t^{\prime}\right)\right] .
\end{aligned}
$$

In the above expressions, $R$ and $\hat{R}$ are coarse-grained forms of $r-v t$ and $i \hat{r}$, respectively. $F$ is adjusted to satisfy the condition $\langle R\rangle=0$. The function $C(v \tau)$ is initially the connected mean-field correlation function $\left\langle\left(r_{M F}(t) r_{M F}(t+\tau)\right\rangle_{c}\right.$.

Ignoring the $R$-dependent terms in the argument of $C$, the action becomes Gaussian, and is invariant under a scale transformation $x \rightarrow b x, t \rightarrow b^{\sigma} t$, $R \rightarrow b^{\sigma-d / 2} R, \hat{R} \rightarrow b^{-\sigma-d / 2} \hat{R}, F \rightarrow b^{-d / 2} F$, and $v \rightarrow b^{-d / 2} v$. Other terms in the action, of higher order in $R$ and $\hat{R}$, that result from the expansion of $C$ [and other terms not explicitly shown in Eq.(14)], decay away at large length and time scales if $d>d_{c}=2 \sigma$. For $d>d_{c}$, the interface is smooth $\left(\zeta_{0}<0\right)$ 
at long length scales, and the depinning exponents take the Gaussian values $z_{0}=\sigma, \nu_{0}=2 / d, \beta_{0}=1$.

At $d=d_{c}$, the action $S$ has an infinite number of marginal terms that can be rearranged as a Taylor series for the function $C\left[v t-v t^{\prime}+R(\mathbf{x}, t)-R\left(\mathbf{x}, t^{\prime}\right)\right]$, when $v \rightarrow 0$. The RG is carried out by integrating over a momentum shell $\Lambda / b<|\mathbf{q}|<\Lambda$ (we set the cutoff wave vector to $\Lambda=1$ for simplicity) and all frequencies, followed by a scale transformation $x \rightarrow b x, t \rightarrow b^{z} t, R \rightarrow b^{\zeta} R$, and $\hat{R} \rightarrow b^{\theta-d} \hat{R}$, where $b=e^{\ell}$. The resulting recursion relation for the linear part in the effective action (to all orders in perturbation theory) is

$$
\frac{\partial\left(F-F_{M F}\right)}{\partial \ell}=(z+\theta)\left(F-F_{M F}\right)+\text { constant },
$$

which immediately implies (with a suitable definition of $F_{c}$ )

$$
\frac{\partial\left(F-F_{c}\right)}{\partial \ell}=y_{F}\left(F-F_{c}\right)
$$

with the exponent identity

$$
y_{F}=z+\theta=1 / \nu .
$$

The functional renormalization of $C(u)$ in $d=2 \sigma-\epsilon$ interface dimensions, computed to one-loop order, gives the recursion relation,

$$
\begin{aligned}
\frac{\partial C(u)}{\partial \ell}=[ & +2 \theta+2(z-\sigma)] C(u)+\zeta u \frac{d C(u)}{d u} \\
& -\frac{S_{d}}{(2 \pi)^{d}} \frac{d}{d u}\left\{[C(u)-C(0)] \frac{d C(u)}{d u}\right\},
\end{aligned}
$$

where $S_{d}$ is the surface area of a unit sphere in $d$ dimensions. NF showed that all higher order diagrams contribute to the renormalization of $C$ as total derivatives with respect to $u$, thus, integrating Eq.(18) at the fixed-point solution $\partial C^{*} / \partial \ell=0$, together with Eqs.(9) and (17), gives $\zeta=\epsilon / 3$ to all orders in $\epsilon$, provided that $\int C^{*} \neq 0$. This gives Eq.(10) for a one-dimensional interface, as argued earlier. This is a consequence of the fact that $C(u)$ remains short-ranged upon renormalization, implying the absence of anomalous contributions to $\zeta$.

The dynamical exponent $z$ is calculated through the renormalization of $\rho$, the term proportional to $\hat{R} \partial_{t} R$, which yields

$$
z=\sigma-2 \epsilon / 9+O\left(\epsilon^{2}\right)
$$


and using the exponent identity (6),

$$
\beta=1-2 \epsilon / 9 \sigma+O\left(\epsilon^{2}\right) .
$$

Nattermann et. al. ${ }^{7}$ obtain the same results to $O(\epsilon)$ by directly averaging the MSR generating function in Eq.(11), and expanding perturbatively around a rigidly moving interface.

Numerical integration of Eq.(1) for an elastic interface ${ }^{11}(\sigma=2)$ has yielded critical exponents $\zeta=0.97 \pm 0.05$ and $\nu=1.05 \pm 0.1$, in agreement with the theoretical result $\zeta=\nu=1$. The velocity exponent $\beta=0.24 \pm 0.1$ is also consistent with the one-loop theoretical result $1 / 3$; however, a logarithmic dependence $v \sim 1 / \ln \left(F-F_{c}\right)$, which corresponds to $\beta=0$, also describes the numerical data well. In contrast, experiments and various discrete models of interface growth have resulted in scaling behaviors that differ from system to system. A number of different experiments on fluid invasion in porous media ${ }^{12}$ give roughness exponents of around 0.8 , while imbibition experiments ${ }^{13,14}$ have resulted in $\zeta \approx 0.6$. A discrete model studied by Leschhorn ${ }^{15}$, motivated by Eq.(1) with $\sigma=2$, gives a roughness exponent of 1.25 at threshold. Since the expansion leading to Eq.(1) breaks down when $\zeta$ approaches one, it is not clear how to reconcile the results of Leschhhorn's numerical work ${ }^{15}$ with the coarsegrained description of the RG calculation, especially since any model with $\zeta>1$ cannot have a coarse grained description based on gradient expansions.

\subsection{Anisotropy}

Amaral, Barabasi, and Stanley $(\mathrm{ABS})^{16}$ recently pointed out that various models of interface depinning in $1+1$ dimensions fall into two distinct classes, depending on the tilt dependence of the interface velocity:

1. For models like the random field Ising Model $^{17}$, and some Solid On Solid models, the computed exponents are consistent with the exponents given by the $\mathrm{RG}$ analysis. It has been suggested ${ }^{15}$, however, that the roughness exponent is systematically larger than $\epsilon / 3$, casting doubt on the exactness of the $R G$ result.

2. A number of different models, based on directed percolation (DP $)^{18,13}$ give a different roughness exponent, $\zeta \approx 0.63$. In these models, pinning sites are randomly distributed with a probability $p$, which is linearly related to the force $F$. The interface is stopped by the boundary of a DP cluster of pinning sites. The critical exponents at depinning can then be related to the longitudinal and transverse correlation length exponents $\nu_{\|} \approx 1.70$ and $\nu_{\perp} \approx 1.07$ of DP. In particular, $\zeta=\nu_{\|} / \nu_{\perp} \approx 0.63$, and $\beta=\nu_{\|}-\nu_{\perp} \approx 0.63$, in agreement with experiments.

The main difference of these models can be understood in terms of the dependence of the threshold force $F_{c}$ to the orientation. To include the possible 
dependence of the line mobility on its slope, $\partial_{x} r$, we can generalize the equation of motion to

$$
\partial_{t} r=K \partial_{x}^{2} r+\kappa \partial_{x} r+\frac{\lambda}{2}\left(\partial_{x} r\right)^{2}+F+f(x, r) .
$$

The isotropic depinning studied by RG corresponds to $\kappa=\lambda=0$. The usual mechanisms for generating a non-zero $\lambda$ are of kinematic origin ${ }^{19}(\lambda \propto v)$ and can be shown to be irrelevant at the depinning threshold where the velocity $v$ goes to zero ${ }^{10}$. However, if $\lambda$ is not proportional to $v$ and stays finite at the transition, it is a relevant operator and expected to modify the critical behavior. As we shall argue below, anisotropy in the medium is a possible source of the nonlinearity at the depinning transition.

A model flux line (FL) confined to move in a plane ${ }^{11,20}$ provides an example where both mechanisms for the nonlinearity are present. Only the force normal to the FL is responsible for motion, and is composed of three components: (1) A term proportional to curvature arising from the smoothening effects of line tension. (2) The Lorentz force due to a uniform current density perpendicular to the plane acts in the normal direction and has a uniform magnitude $F$ (per unit line length). (3) A random force $\hat{\mathbf{n}} \cdot \mathbf{f}$ due to impurities, where $\hat{\mathbf{n}}$ is the unit normal vector ${ }^{20}$. Equating viscous dissipation with the work done by the normal force leads to the equation of motion

$$
\frac{\partial h}{\partial t}=\sqrt{1+s^{2}}\left[\frac{\partial_{x}^{2} h}{\left(1+s^{2}\right)^{3 / 2}}+F+\frac{f_{h}-s f_{x}}{\sqrt{1+s^{2}}}\right],
$$

where $h(x, t)$ denotes transverse displacement of the line and $s \equiv \partial_{x} h$. The nonlinearities generated by $\sqrt{1+s^{2}}$ are kinematic in origin ${ }^{19}$ and irrelevant as $v \rightarrow 0^{10}$, as can be seen easily by taking them to the left hand side of Eq.(22). The shape of the pinned FL is determined by the competition of the terms in the square brackets. Although there is no explicit simple $s^{2}$ term in this group, it will be generated if the system is anisotropic.

To illustrate the idea, let us take $f_{h}$ and $f_{x}$ to be independent random fields with amplitudes $\Delta_{h}^{1 / 2}$ and $\Delta_{x}^{1 / 2}$ respectively; each correlated isotropically in space within a distance $a$. For weak disorder, a deformation of order $a$ in the normal direction $\hat{\mathbf{n}}$ takes place over a distance $L_{c} \gg a$ along the line. The total force due to curvature on this piece of the line is of the order of $L_{c}\left(a / L_{c}^{2}\right)$, and the pinning force, $\left[\left(L_{c} / a\right)\left(n_{h}^{2} \Delta_{h}+n_{x}^{2} \Delta_{x}\right)\right]^{1 / 2}$. Equating the two forces ${ }^{2}$ yields $L_{c}=a\left(n_{h}^{2} \Delta_{h}+n_{x}^{2} \Delta_{x}\right)^{-1 / 3}$ and an effective pinning strength per unit length,

$$
F_{0}(s)=a L_{c}^{-2}=a^{-1}\left(\frac{\Delta_{h}+s^{2} \Delta_{x}}{1+s^{2}}\right)^{2 / 3} .
$$

The roughening by impurities thus reduces the effective driving force on the scale $L_{c}$ to $\tilde{F}(s)=F-F_{0}(s)$. Therefore, even if initially $F$ is independent 
of $s$, such a dependence is generated under coarse graining, provided that the random force is anisotropic, i.e. $\Delta_{h} \neq \Delta_{x}$. An expansion of $\tilde{F}(s)$ around its maximum (which defines the hard direction) yields an $s^{2}$ term which is positive and remains finite as $v \rightarrow 0$.

The above example indicates the origin of the two types of behavior for $\lambda_{\text {eff }}=v^{\prime \prime}(s=0)$ observed by $\mathrm{ABS}^{16}$ : Kinematics produces a $\lambda_{\text {eff }}$ proportional to $v$ which vanishes at the threshold; anisotropy yields a nonvanishing (and diverging) $\lambda_{\text {eff }}$ at the depinning transition. An immediate consequence of the latter is that the depinning threshold $F_{c}$ depends on the average orientation of the line. While anisotropy may generate other local terms in the effective equation of motion, at a symmetry direction, this term is the only relevant one in the RG sense, capable of modifying the critical behavior for $d \leq 4$. A oneloop RG of Eq.(21) with the $\kappa=0$ was carried out by Stepanow ${ }^{21}$. He finds no stable fixed point for $2 \leq d \leq 4$, but his numerical integration of the one loop RG equations in $d=1$ yield $\zeta \approx 0.8615$ and a dynamical exponent $z=1$. Due to the absence of Galilean invariance, there is also a renormalization of $\lambda$ which is related to the diverging $\lambda_{\text {eff }}$ observed in Ref. ${ }^{16}$. The nonperturbative nature of the fixed point precludes a gauge of the reliability of these exponents.

Numerical simulations of Eq.(21) in $d=1^{22}$, indicate that it shares the characteristics of a class of lattice models ${ }^{18,13}$ where the external force is related to the density $p$ of "blocking sites" by $F=1-p$. When $p$ exceeds a critical value of $p_{c}$, blocking sites form a directed percolating path which stops the interface. For a given geometry, there is a direction along which the first spanning path appears. This defines a hard direction for depinning where the threshold force $F_{c}(s)$ reaches maximum. Higher densities of blocking sites are needed to form a spanning path away from this direction, resulting in a lower threshold force $F_{c}(s)$ for a tilted interface. Thus on a phenomenological level we believe that the nonlinear equation, and directed percolation (DP) models of interface depinning belong to the same universality class of anisotropic depinning. This analogy may in fact be generalized to higher dimensions, where the blocking path is replaced by a directed blocking surface ${ }^{23}$. Unfortunately, little is known analytically about the scaling properties of such a surface at the percolation threshold.

As emphasized above, the hallmark of anisotropic depinning is the dependence of the threshold force $F_{c}(s)$ on the slope $s$. Above this threshold, we expect $v(F, s)$ to be an analytical function of $F$ and $s$. In particular, for $F>F_{c}(0)$, there is a small $s$ expansion $v(F, s)=v(F, s=0)+\lambda_{\text {eff }} s^{2} / 2+\cdots$. On the other hand, we can associate a characteristic slope $\bar{s}=\xi_{\perp} / \xi_{\|} \sim(\delta F)^{\nu(1-\zeta)}$, to DP clusters where $\delta F=F-F_{c}(0)$, and $\nu$ is the correlation length exponent. Scaling then suggests

$$
v(F, s)=(\delta F)^{\theta} g\left(s / \delta F^{\nu(1-\zeta)}\right),
$$


where $\theta=\nu(z-\zeta)$. Matching Eq.(23) with the small $s$ expansion, we see that $\lambda_{\text {eff }}$ diverges as $(\delta F)^{-\phi}$ (as defined by $\mathrm{ABS}^{16}$ ) with $\phi=2 \nu(1-\zeta)-\theta=$ $\nu(2-\zeta-z)$. In $d=1$, the exponents $\nu$ and $\zeta$ are related to the correlation length exponents $\nu_{\|}$and $\nu_{\perp}$ of $\mathrm{DP}^{23}$ via $\nu=\nu_{\|} \approx 1.73$ and $\zeta=\nu_{\perp} / \nu_{\|} \approx 0.63$, while the dynamical exponent is $z=1$. Scaling thus predicts $\phi \approx 0.63$, in agreement with the numerical result of $0.64 \pm 0.08$ in Ref. ${ }^{16}$. Close to the line $F=F_{c}(0)$ (but at a finite $s$ ), the dependence of $v$ on $\delta F$ drops out and we have

$$
v\left(F_{c}, s\right) \propto|s|^{\theta / \nu(1-\zeta)} .
$$

As $z=1$ in $d=1$, the above equation reduces to $v \propto|s|$, in agreement with Fig. 1 of Ref. ${ }^{16}$. Since $v(F, s)=0$ at $F=F_{c}(s)$, Eq.(23) suggests

$$
F_{c}(s)-F_{c}(0) \propto-|s|^{1 / \nu(1-\zeta)} .
$$

Note that Eqs. (24) and (25) are valid also in higher dimensions, though values of the exponents quoted above vary with $d^{23}$.

An interface tilted away from the hard direction not only has a different depinning threshold, but also completely different scaling behavior at its transition. This is because, due to the presence of an average interface gradient $\mathbf{s}=\langle\nabla h\rangle$, the isotropy in the internal $\mathbf{x}$ space is lost. The equation of motion for fluctuations, $h^{\prime}(\mathbf{x}, t)=h(\mathbf{x}, t)-\mathbf{s} \cdot \mathbf{x}$, around the average interface position may thus include a non-zero $\kappa$ in $(21)$. The resulting depinning transition belongs to yet a new universality class with anisotropic response and correlation functions in directions parallel and perpendicular to s; i.e.

$$
\begin{aligned}
\left\langle\left[h(\mathbf{x})-h\left(\mathbf{x}^{\prime}\right)\right]^{2}\right\rangle & =\left|x_{\|}-x_{\|}^{\prime}\right|^{\zeta} \mathcal{F}\left(\frac{\left|\mathbf{x}_{\mathbf{t}}-\mathbf{x}_{\mathbf{t}}^{\prime}\right|}{\left|x_{\|}-x_{\|}^{\prime}\right|^{\eta}}\right) \\
& \rightarrow \begin{cases}\left|x_{\|}-x_{\|}^{\prime}\right|^{\zeta} & \text { for } \mathbf{x}_{\mathbf{t}}-\mathbf{x}_{\mathbf{t}}^{\prime}=0 \\
\left|\mathbf{x}_{\mathbf{t}}-\mathbf{x}_{\mathbf{t}}^{\prime}\right|^{\zeta / \eta} & \text { for } x_{\|}-x_{\|}^{\prime}=0\end{cases}
\end{aligned}
$$

where $\eta$ is the ansiotropy exponent, and $\mathbf{x}_{t}$ denotes the $d-1$ directions transverse to $\mathbf{s}$.

A suggestive mapping allows us to determine the exponents for depinning a tilted interface: Consider the response to a perturbation in which all points along a $(d-1)$-dimensional cross section of the interface at a fixed $x_{\|}$are pushed up by a small amount. This move decreases the slope of the interface uphill but increases it downhill. Since $F_{c}(s)$ decreases with increasing $s$, at criticality the perturbation propagates only a finite distance uphill but causes a downhill avalanche. The disturbance front moves at a constant velocity $\left(\delta x_{\|} \propto t\right)$ and hence $z_{\|}=1$. (Such chains of moving sites were indeed seen in simulations of the $d=2$ model discussed below.) Furthermore, the evolution of successive 
cross sections $\mathbf{x}_{t}\left(x_{\|}\right)$is expected to be the same as the evolution in time of a $(d-1)$-dimensional interface! The latter is governed by the Kardar-ParisiZhang (KPZ) equation ${ }^{19}$, whose scaling behavior has been extensively studied. From this analogy we conclude,

$$
\zeta(d)=\frac{\zeta_{\mathrm{KPZ}}(d-1)}{z_{\mathrm{KPZ}}(d-1)}, \quad \eta(d)=\frac{1}{z_{\mathrm{KPZ}}(d-1)} .
$$

In particular, the tilted interface with $d=2$ maps to the growth problem in $1+1$ dimensions where the exponents are known exactly, yielding $\zeta(2)=1 / 3$ and $\eta(2)=2 / 3$. This picture can be made more precise for a lattice model introduced below. Details will be presented elsewhere.

To get the exponent $\beta$ for the vanishing of velocity of the tilted interface, we note that since $z_{\|}=1, v$ scales as the excess slope $\delta s=s-s_{c}(F)$. The latter controls the density of the above moving fronts; $s_{c}(F)$ is the slope of the critical interface at a given driving force $F$, i.e., $F=F_{c}\left(s_{c}\right)$. Away from the symmetry direction, the function $F_{c}(s)$ has a non-vanishing derivative and hence

$$
\delta F=F-F_{c}(s)=F_{c}\left(s_{c}\right)-F_{c}(s) \sim \delta s \sim v .
$$

We thus conclude that generically $\beta=1$ for tilted interfaces, independent of dimension.

To check the above predictions, we performed simulations of the parallelized version of a previously studied percolation model of interface depinning ${ }^{18}$. A solid-on-solid (SOS) interface is described by a set of integer heights $\left\{h_{\mathbf{i}}\right\}$ where $\mathbf{i}$ is a group of $d$ integers. With each configuration is associated a random set of pinning forces $\left\{\eta_{\mathbf{i}} \in[0,1)\right\}$. The heights are updated in parallel according to the following rules: $h_{\mathbf{i}}$ is increased by one if (i) $h_{\mathbf{i}} \leq h_{\mathbf{j}}-2$ for at least one $\mathbf{j}$ which is a nearest neighbor of $\mathbf{i}$, or (ii) $\eta_{\mathbf{i}}<F$ for a pre-selected uniform force $F$. If $h_{\mathbf{i}}$ is increased, the associated random force $\eta_{\mathbf{i}}$ is also updated, i.e. replaced by a new random number in the interval $[0,1)$. Otherwise, $h_{\mathbf{i}}$ and $\eta_{\mathbf{i}}$ are unchanged. The simulation is started with initial conditions $h_{\mathbf{i}}(t=0)=\operatorname{Int}\left[s \mathbf{i}_{x}\right]$, and boundary conditions $h_{\mathbf{i}+\mathbf{L}}=\operatorname{Int}[s L]+h_{\mathbf{i}}$ are enforced throughout. The CPU time is greatly reduced by only keeping track of active sites.

The above model has a simple analogy to a resistor-diode percolation problem $^{23}$. Condition (i) ensures that, once a site $(\mathbf{i}, h)$ is wet (i.e., on or behind the interface), all neighboring columns of $\mathbf{i}$ must be wet up to height $h-1$. Thus there is always "conduction" from a site at height $h$ to sites in the neighboring columns at height $h-1$. This relation can be represented by diodes pointing diagonally downward. Condition (ii) implies that "conduction" may also occur upward. Hence a fraction $F$ of vertical bonds are turned into resistors which allow for two-way conduction. Note that, due to the SOS 
condition, vertical downward conduction is always possible. For $F<F_{c}$, conducting sites connected to a point lead at the origin, form a cone whose hull is the interface separating wet and dry regions. The opening angle of the cone increases with $F$, reaching $180^{\circ}$ at $F=F_{c}$, beyond which percolation in the entire space takes place, so that all sites are eventually wet. If instead of a point, we start with a planar lead defining the initial surface, the percolation threshold depends on the surface orientation, with the highest threshold for the untilted one.

Our simulations of lattices of 65536 sites in $d=1$ and of $512 \times 512$ and $840 \times 840$ sites in $d=2$ confirm the exponents for depinning in the hard direction. For a tilted surface in $d=1$ the roughness exponent determined from the height-height correlation function is consistent with the predicted value of $\zeta=1 / 2$ and different from $\zeta \approx 0.63$ of the untilted one. The dependence of the depinning threshold on slope is clearly seen in the figure below, where the average velocity is plotted against the driving force for $s=0$ (open) and $s=1 / 2$ (solid). The $s=0$ data can be fitted to a power-law $v \sim\left(F-F_{c}\right)^{\theta}$, where $F_{c} \approx 0.461, \beta=0.63 \pm 0.04$ for $d=1$, and $F_{c} \approx 0.201, \beta=0.72 \pm 0.04$ for $d=2$. Data at $s=1 / 2$ are consistent with Eq.(27) close to the threshold.

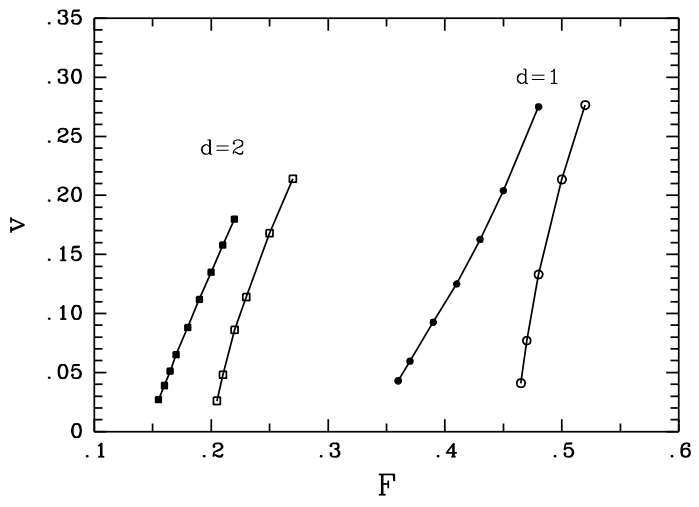

Figure 3. Average interface velocity $v$ versus the driving force $F$, for $d=1, s=0$ (open circles), $d=1, s=1 / 2$ (solid circles), $d=2$, $s=0$ (open squares), and $d=2, s=1 / 2$ (solid squares).

We also measured height-height correlation functions at the depinning transition. For a tilted surface in $d=2$, the height fluctuations and corresponding dynamic behaviors are different parallel and transverse to the tilt. The next figure shows a scaling plot of (a) $C_{\|}\left(r_{\|}, t\right) \equiv\left\langle\left[h\left(x_{\|}+r_{\|}, x_{t}, t\right)-h\left(x_{\|}, x_{t}, t\right)\right]^{2}\right\rangle$ and (b) $C_{t}\left(r_{t}, t\right) \equiv\left\langle\left[h\left(x_{\|}, x_{t}+r_{t}, t\right)-h\left(x_{\|}, x_{t}, t\right)\right]^{2}\right\rangle$ against the scaled distances at the depinning threshold of an $s=1 / 2$ interface. Each curve shows data at 
a given $t=32,64, \cdots, 1024$, averaged over 50 realizations of the disorder. The data collapse is in agreement with the mapping to the KPZ equation in one less dimension.
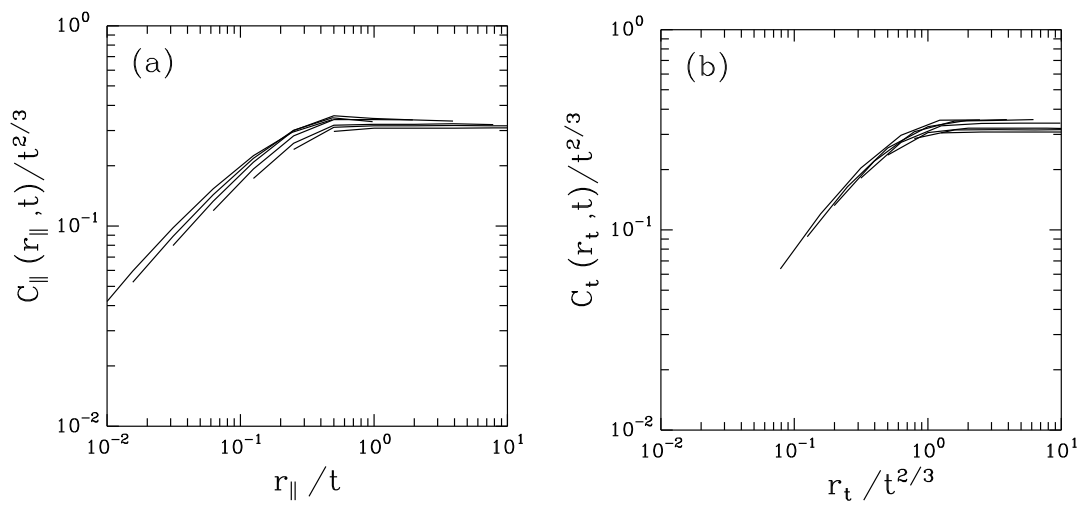

Figure 4. Height-height correlation functions (a) along and (b) transverse to the tilt for an $840^{2}$ system at different times $32 \leq t \leq$ 1024. The interface at $t=0$ is flat; $d=2, s=1 / 2$, and $F=0.144$.

In summary, critical behavior at the depinning of an interface depends on the symmetries of the underlying medium. Different universality classes can be distinguished from the dependence of the threshold force (or velocity) on the slope, which is reminiscent of similar dependence in a model of resistor-diode percolation. In addition to isotropic depinning, we have so far identified two classes of anisotropic depinning: along a (hard) axis of inversion symmetry in the plane, and tilted away from it. We have no analytical results in the former case, but suggest a number of scaling relations that are validated by simulations. In the latter (more generic) case we have obtained exact information from a mapping to moving interfaces, and confirmed them by simulations in $d=1$ and $d=2$. As it is quite common to encounter (intrinsic or artificially fabricated) anisotropy for flux lines in superconductors, domain walls in magnets, and interfaces in porous media, we expect our results to have important experimental ramifications.

Another form of anisotropy is also possible for interfaces in $2+1$ dimensions. If the directions $x$ and $y$ on the surface are not related by symmetry, the non-linear term in the KPZ equation can be generalized, leading to the depinning equation

$$
\partial_{t} h=K_{x} \partial_{x}^{2} r+K_{y} \partial_{x}^{2} r+\frac{\lambda_{x}}{2}\left(\partial_{x} r\right)^{2}+\frac{\lambda_{y}}{2}\left(\partial_{y} r\right)^{2}+F+f(x, y, r) .
$$


In fact the difference between $K_{x}$ and $K_{y}$ is not important as long as both are positive. It was first pointed out by Dietrich Wolf ${ }^{24}$ that different signs of $\lambda_{x}$ and $\lambda_{y}$ lead to a different universality class for the case of annealed noise. More recently it was demonstrated by Jeong et $\mathrm{al}^{25}$ that, with quenched noise, eq.(28)describes a new universality class of depinning transitions with $\beta \approx 0.80(1)$, and anisotropic roughness exponents in the $x$ and $y$ directions.

\section{Fluctuating Lines}

\subsection{Flux Line Depinning}

The pinning of flux lines (FLs) in Type-II superconductors is of fundamental importance to many technological applications that require large critical currents $^{26}$. Upon application of an external current density $J$, the motion of FLs due to the Lorentz force causes undesirable dissipation of supercurrents. Major increases in the critical current density $J_{c}$ of a sample are achieved when the FLs are pinned to impurities. There are many recent studies, both experimental ${ }^{27,28}$ and theoretical ${ }^{29,30}$, on collective pinning of FL's to point or columnar defects. Another consequence of impurities is the strongly nonlinear behavior of the current slightly above the depinning threshold, as the FLs start to move across the sample. Recent numerical simulations have concentrated

on the low temperature behavior of a single FL near depinning ${ }^{31,11,20}$, mostly ignoring fluctuations transverse to the plane defined by the magnetic field and the Lorentz force. Common signatures of the depinning transition from $J<J_{c}$ to $J>J_{c}$ include a broad band ( $f^{-a}$ type) voltage noise spectrum, and selfsimilar fluctuations of the FL profile.

The FL provides yet another example of a depinning transition. We now extend the methods of the previous section to the full three-dimensional dynamics of a single FL at low temperatures. The shape of the FL at a given time $t$ is described by $\mathbf{r}(x, t)$, where $x$ is along the magnetic field $B$, and the unit vector $\mathbf{e}_{\|}$is along the Lorentz force $\mathbf{F}$. Point impurities are modeled by a random potential $V(x, \mathbf{r})$, with zero mean and short-range correlations. In the presence of impurities and a bulk Lorentz force $\mathbf{F}$, the energy of a FL with small fluctuations is,

$$
\mathcal{H}=\int d x\left\{\frac{1}{2}\left(\partial_{x} \mathbf{r}\right)^{2}+V(x, \mathbf{r}(x, t))-\mathbf{r}(x, t) \cdot \mathbf{F}\right\} .
$$

The simplest possible Langevin equation for the FL, consistent with local, dissipative dynamics, is

$$
\mu^{-1} \frac{\partial \mathbf{r}}{\partial t}=-\frac{\delta \mathcal{H}}{\delta \mathbf{r}}=\partial_{x}^{2} \mathbf{r}+\mathbf{f}(x, \mathbf{r}(x, t))+\mathbf{F},
$$




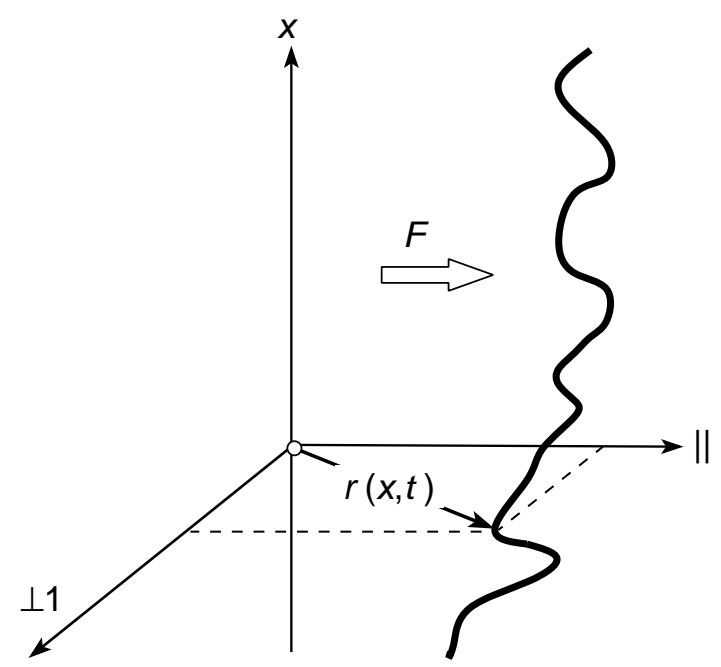

Figure 5. Geometry of the line in three dimensions.

where $\mu$ is the mobility of the FL, and $\mathbf{f}=-\nabla_{\mathbf{r}} V$. The potential $V(x, \mathbf{r})$ need not be isotropic. For example, in a single crystal of ceramic superconductors with the field along the oxide planes, it will be easier to move the FL along the planes. This leads to a pinning threshold that depends on the orientation of the force. Anisotropy also modifies the line tension, and the elastic term in Eq.(30) is in general multiplied by a non-diagonal matrix $K_{\alpha \beta}$. The random force $\mathbf{f}(x, \mathbf{r})$, can be taken to have zero mean with correlations

$$
\left\langle f_{\alpha}(x, \mathbf{r}) f_{\gamma}\left(x^{\prime}, \mathbf{r}^{\prime}\right)\right\rangle=\delta\left(x-x^{\prime}\right) \Delta_{\alpha \gamma}\left(\mathbf{r}-\mathbf{r}^{\prime}\right) .
$$

We shall focus mostly on the isotropic case, with $\Delta_{\alpha \gamma}\left(\mathbf{r}-\mathbf{r}^{\prime}\right)=\delta_{\alpha \gamma} \Delta\left(\left|\mathbf{r}-\mathbf{r}^{\prime}\right|\right)$, where $\Delta$ is a function that decays rapidly for large values of its argument.

While the flux line is pinned by impurities when $F<F_{c}$, for $F$ slightly above threshold, we expect the average velocity $v=|\mathbf{v}|$ to scale as in Eq.(23). Superposed on the steady advance of the FL are rapid "jumps" as portions of the line depin from strong pinning centers. The cut off length $\xi$ on avalanche sizes diverges on approaching the threshold as $\xi \sim\left(F-F_{c}\right)^{-\nu}$. At length scales up to $\xi$, the correlated fluctuations satisfy the dynamic scaling forms,

$$
\begin{aligned}
\left\langle\left[r_{\|}(x, t)-r_{\|}(0,0)\right]^{2}\right\rangle & =|x|^{2 \zeta_{\|}} g_{\|}\left(t /|x|^{z_{\|}}\right), \\
\left\langle\left[r_{\perp}(x, t)-r_{\perp}(0,0)\right]^{2}\right\rangle & =|x|^{2 \zeta_{\perp}} g_{\perp}\left(t /|x|^{z_{\perp}}\right),
\end{aligned}
$$

where $\zeta_{\alpha}$ and $z_{\alpha}$ are the roughness and dynamic exponents, respectively. The scaling functions $g_{\alpha}$ go to a constant as their arguments approach 0. Beyond 
the length scale $\xi$, different regions of the FL depin more or less independently and the system crosses over to a moving state, described by different exponents, which will be considered in the next section.

The major difference of this model from the previously studied interface is that the position of the flux line, $\mathbf{r}(x, t)$, is now a 2 -dimensional vector instead of a scalar; fluctuating along both $\mathbf{e}_{\|}$and $\mathbf{e}_{\perp}$ directions. One consequence is that a "no passing" rule ${ }^{32}$, applicable to CDWs and interfaces, does not apply to FLs. It is possible to have coexistence of moving and stationary FLs in particular realizations of the random potential. How do these transverse fluctuations scale near the depinning transition, and do they in turn influence the critical dynamics of longitudinal fluctuations near threshold? The answer to the second question can be obtained by the following qualitative argument: Consider Eq.(30) for a particular realization of randomness $\mathbf{f}(x, \mathbf{r})$. Assuming that portions of the FL always move in the forward direction, there is a unique point $r_{\perp}\left(x, r_{\|}\right)$that is visited by the line for given coordinates $\left(x, r_{\|}\right)$. We construct a new force field $f^{\prime}$ on a two dimensional space $\left(x, r_{\|}\right)$ through $f^{\prime}\left(x, r_{\|}\right) \equiv f_{\|}\left(x, r_{\|}, r_{\perp}\left(x, r_{\|}\right)\right)$. It is then clear that the dynamics of the longitudinal component $r_{\|}(x, t)$ in a given force field $\mathbf{f}(x, \mathbf{r})$ is identical to the dynamics of $r_{\|}(x, t)$ in a force field $f^{\prime}\left(x, r_{\|}\right)$, with $r_{\perp}$ set to zero. It is quite plausible that, after averaging over all $\mathbf{f}$, the correlations in $f^{\prime}$ will also be short-ranged, albeit different from those of $\mathbf{f}$. Thus, the scaling of longitudinal fluctuations of the depinning FL will not change upon taking into account transverse fluctuations. However, the question of how these transverse fluctuations scale still remains.

Certain statistical symmetries of the system restrict the form of response and correlation functions. For example, Eq.(30) has statistical space- and timetranslational invariance, which enables us to work in Fourier space, i.e. $(x, t) \rightarrow$ $(q, \omega)$. For an isotropic medium, $\mathbf{F}$ and $\mathbf{v}$ are parallel to each other, i.e., $\mathbf{v}(\mathbf{F})=$ $v(F) \hat{\mathbf{F}}$, where $\hat{\mathbf{F}}$ is the unit vector along $\mathbf{F}$. Furthermore, all expectation values involving odd powers of a transverse component are identically zero due to the statistical invariance under the transformation $r_{\perp} \rightarrow-r_{\perp}$. Thus, linear response and two-point correlation functions are diagonal. The introduced critical exponents are then related through scaling identities. These can be derived from the linear response to an infinitesimal external force field $\varepsilon(q, \omega)$,

$$
\chi_{\alpha \beta}(q, \omega)=\left\langle\frac{\partial r_{\alpha}(q, \omega)}{\partial \varepsilon_{\beta}(q, \omega)}\right\rangle \equiv \delta_{\alpha \beta} \chi_{\alpha}(q, \omega),
$$

in the $(q, \omega) \rightarrow(0,0)$ limit. Eq.(30) is statistically invariant under the transformation $\mathbf{F} \rightarrow \mathbf{F}+\varepsilon(q), \mathbf{r}(q, \omega) \rightarrow \mathbf{r}(q, \omega)+q^{-2} \varepsilon(q)$. Thus, the static linear response has the form $\chi_{\|}(q, \omega=0)=\chi_{\perp}(q, \omega=0)=q^{-2}$. Since $\varepsilon_{\|}$scales like the applied force, the form of the linear response at the correlation length $\xi$ 
gives the exponent identity

$$
\zeta_{\|}+1 / \nu=2 .
$$

Considering the transverse linear response seems to imply $\zeta_{\perp}=\zeta_{\|}$. However, the static part of the transverse linear response is irrelevant at the critical RG fixed point, since $z_{\perp}>z_{\|}$, as shown below. When a slowly varying uniform external force $\varepsilon(t)$ is applied, the FL responds as if the instantaneous external force $\mathbf{F}+\varepsilon$ is a constant, acquiring an average velocity,

$$
\left\langle\partial_{t} r_{\alpha}\right\rangle=v_{\alpha}(\mathbf{F}+\varepsilon) \approx v_{\alpha}(\mathbf{F})+\frac{\partial v_{\alpha}}{\partial F_{\gamma}} \varepsilon_{\gamma} .
$$

Substituting $\partial v_{\|} / \partial F_{\|}=d v / d F$ and $\partial v_{\perp} / \partial F_{\perp}=v / F$, and Fourier transforming, gives

$$
\begin{aligned}
\chi_{\|}(q=0, \omega) & =\frac{1}{-i \omega(d v / d F)^{-1}+O\left(\omega^{2}\right)}, \\
\chi_{\perp}(q=0, \omega) & =\frac{1}{-i \omega(v / F)^{-1}+O\left(\omega^{2}\right)} .
\end{aligned}
$$

Combining these with the static response, we see that the characteristic relaxation times of fluctuations with wavelength $\xi$ are

$$
\begin{aligned}
& \tau_{\|}\left(q=\xi^{-1}\right) \sim\left(q^{2} \frac{d v}{d F}\right)^{-1} \sim \xi^{2+(\beta-1) / \nu} \sim \xi^{z_{\|}}, \\
& \tau_{\perp}\left(q=\xi^{-1}\right) \sim\left(q^{2} \frac{v}{F}\right)^{-1} \sim \xi^{2+\beta / \nu} \sim \xi^{z_{\perp}}
\end{aligned}
$$

which, using Eq.(34), yield the scaling relations

$$
\begin{aligned}
& \beta=\left(z_{\|}-\zeta_{\|}\right) \nu, \\
& z_{\perp}=z_{\|}+1 / \nu .
\end{aligned}
$$

We already see that the dynamic relaxation of transverse fluctuations is much slower than longitudinal ones. All critical exponents can be calculated from $\zeta_{\|}, \zeta_{\perp}$, and $z_{\|}$, by using $\operatorname{Eqs}(34)$, and (36).

Equation (30) can be analyzed using the formalism of Martin, Siggia, and Rose $(\mathrm{MSR})^{9}$. Ignoring transverse fluctuations, and generalizing to $d$ dimensional internal coordinates $\mathbf{x} \in \Re^{d}$, leads to an interface depinning model which was studied by Nattermann, Stepanow, Tang, and Leschhorn (NSTL) ${ }^{7}$, and by Narayan and Fisher $(\mathrm{NF})^{10}$. The RG treatment indicates that impurity disorder becomes relevant for dimensions $d \leq 4$, and the critical exponents in $d=4-\epsilon$ dimensions are given to one-loop order as $\zeta=\epsilon / 3, z=2-2 \epsilon / 9$. NSTL obtained this result by directly averaging the MSR generating functional $Z$, 
and calculating the renormalization of the force-force correlation function $\Delta(r)$, perturbatively around the freely moving interface $[\Delta(r)=0]$. NF, on the other hand, used a perturbative expansion of $Z$, around a saddle point corresponding to a mean-field approximation to Eq. $(30)^{33}$, which involved temporal force-force correlations $C(v t)$. They argue that a conventional low-frequency analysis is not sufficient to determine critical exponents. They also suggest that the roughness exponent is equal to $\epsilon / 3$ to all orders in perturbation theory.

Following the approach of NF, we employ a perturbative expansion of the disorder-averaged MSR partition function around a mean-field solution for cusped impurity potentials ${ }^{10}$. All terms in the expansion involving longitudinal fluctuations are identical to the interface case, thus we obtain the same critical exponents for longitudinal fluctuations, i.e., $\zeta_{\|}=\epsilon / 3, z_{\|}=2-2 \epsilon / 9+O\left(\epsilon^{2}\right)$. Furthermore, for isotropic potentials, the renormalization of transverse temporal force-force correlations $C_{\perp}(v t)$ yields a transverse roughness exponent $\zeta_{\perp}=5 \zeta_{\|} / 2-2$, to all orders in perturbation theory. For the FL $(\epsilon=3)$, the critical exponents are then given by

$$
\begin{gathered}
\zeta_{\|}=1, \quad z_{\|} \approx 4 / 3, \quad \nu=1, \\
\beta \approx 1 / 3, \quad \zeta_{\perp}=1 / 2, \quad z_{\perp} \approx 7 / 3 .
\end{gathered}
$$

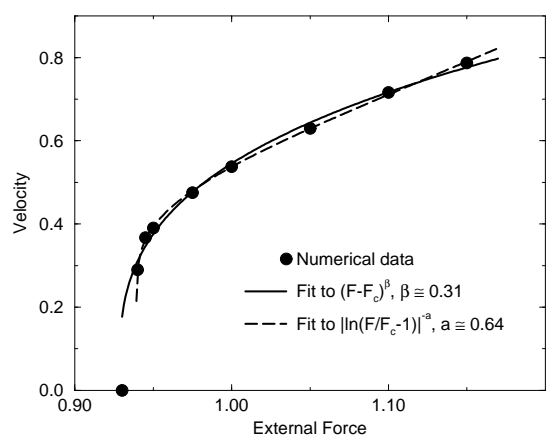

Figure 6. A plot of average velocity versus external force for a system of 2048 points. Statistical errors are smaller than symbol sizes. Both fits have three adjustable parameters: The threshold force, the exponent, and an overall multiplicative constant.

To test the scaling forms and exponents predicted by Eqs.(23) and (32), we numerically integrated Eq.(30), discretized in coordinates $x$ and $t$. Free boundary conditions were used for system sizes of up to 2048, with a grid 


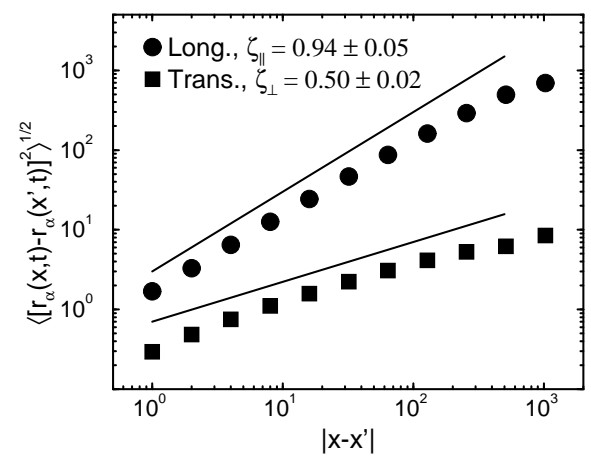

Figure 7. A plot of equal time correlation functions versus separation, for the system shown in Fig.6, at $F=0.95$. The observed roughness exponents very closely follow the theoretical predictions of $\zeta_{\|}=1, \zeta_{\perp}=0.5$, which are shown as solid lines for comparison.

spacing $\Delta x=1$ and a time step $\Delta t=0.02$. Time averages were evaluated after the system reached steady state. Periodic boundary conditions gave similar results, but with larger finite size effects. Smaller grid sizes did not change the results considerably. The behavior of $v(F)$ seems to fit the scaling form of Eq.(23) with an exponent $\beta \approx 0.3$, but is also consistent with a logarithmic dependence on the reduced force, i.e., $\beta=0$. The same behavior was observed by Dong et al. in a recent simulation of the $1+1$ dimensional geometry ${ }^{11}$. Since $z_{\|}$, and consequently $\beta$, is known only to first order in $\epsilon$, higher order corrections are expected. By looking at equal time correlation functions, we find that transverse fluctuations are strongly suppressed, and that the roughness exponents are equal to our theoretical estimates within statistical accuracy. The excellent agreement for $\epsilon=3$ suggests that the theoretical estimates are indeed exact.

The potential pinning the FL in a single superconducting crystal is likely to be highly anisotropic. For example, consider a magnetic field parallel to the copper oxide planes of a ceramic superconductor. The threshold force then depends on its orientation, with depinning easiest along the copper oxide planes. In general, the average velocity may depend on the orientations of the external force and the FL. The most general gradient expansion for the equation of motion is then,

$\frac{\partial r_{\alpha}}{\partial t}=\mu_{\alpha \beta} F_{\beta}+\kappa_{\alpha \beta} \partial_{x} r_{\beta}+K_{\alpha \beta} \partial_{x}^{2} r_{\beta}+\frac{1}{2} \Lambda_{\alpha, \beta \gamma} \partial_{x} r_{\beta} \partial_{x} r_{\gamma}+f_{\alpha}(x, \mathbf{r}(x, t))+\cdots$, 
with

$$
\left\langle f_{\alpha}(x, \mathbf{r}) f_{\beta}\left(x^{\prime}, \mathbf{r}^{\prime}\right)\right\rangle=\delta\left(x-x^{\prime}\right) C_{\alpha \beta}\left(\mathbf{r}-\mathbf{r}^{\prime}\right) .
$$

Depending on the presence or absence of various terms allowed by the symmetries of the system, the above set of equations encompasses many distinct universality classes. For example, consider the situation where $\mathbf{v}$ depends on $\mathbf{F}$, but not on the orientation of the line. Eqs.(35) have to be modified, since $\mathbf{v}$ and $\mathbf{F}$ are no longer parallel (except along the axes with $\mathbf{r} \rightarrow-\mathbf{r}$ symmetry), and the linear response function is not diagonal. The RG analysis is more cumbersome: For depinning along a non-symmetric direction, the longitudinal exponents are not modified (in agreement with the argument presented earlier), while the transverse fluctuations are further suppressed to $\zeta_{\perp}=2 \zeta_{\|}-2$ (equal to zero for $\left.\zeta_{\|}=1\right)^{34}$. Relaxation of transverse modes are still characterized by $z_{\perp}=z_{\|}+1 / \nu$, and the exponent identity (34) also holds. Surprisingly, the exponents for depinning along axes of reflection symmetry are the same as the isotropic case.

If the velocity also depends on the tilt, there will be additional relevant terms in the MSR partition function, which invalidate the arguments leading to Eq.(34). The analogy to FLs in a planes suggests that the longitudinal exponents for $d=1$ are controlled by DP clusters $^{18,13}$, with $\zeta_{\|} \approx 0.63$. Since no perturbative fixed point is present in this case, it is not clear how to explore the behavior of transverse fluctuations systematically.

\subsection{Dynamic Fluctuations of an Unpinned Flux Line}

So far,w investigated the dynamics of a Flux Line near the depinning transition. Now, we would like to consider its behavior in a different regime, when the external driving force is large, and the impurities appear as weak barriers that deflect portions of the line without impeding its overall drift. In such non-equilibrium systems, one can regard the evolution equations as more fundamental, and proceed by constructing the most general equations consistent with the symmetries and conservation laws of the situation under study $^{35}$. Even in a system with isotropic randomness, which we will discuss here, the average drift velocity, $v$, breaks the symmetry between forward and backward motions, and allows introduction of nonlinearities in the equations of motion ${ }^{36,35}$.

Let us first concentrate on an interface in two dimensions. (Fig.1.) By contracting up to two spatial derivatives of $r$, and keeping terms that are relevant, one obtains the Kardar-Parisi-Zhang ${ }^{19}$ (KPZ) equation,

$$
\partial_{t} r(x, t)=\mu F+K \partial_{x}^{2} r(x, t)+\frac{\lambda}{2}\left[\partial_{x} r(x, t)\right]^{2}+f(x, t),
$$

with random force correlations

$$
\left\langle f(x, t) f\left(x^{\prime}, t^{\prime}\right)\right\rangle=2 T \delta\left(x-x^{\prime}\right) \delta\left(t-t^{\prime}\right) .
$$


For a moving line, the term proportional to the external force can be absorbed without loss of generality by considering a suitable Galilean transformation, $r \rightarrow r-a t$, to a moving frame. A large number of stochastic nonequilibrium growth models, like the Eden Model and various ballistic deposition models are known to be well described, at large length scales and times, by this equation, which is intimately related to several other problems. For example, the transformation $v(x, t)=-\lambda \partial_{x} r(x, t)$ maps Eq.(40) to the randomly stirred Burgers' equation for fluid flow ${ }^{37,38}$,

$$
\partial_{t} v+v \partial_{x} v=K \partial_{x}^{2} v-\lambda \partial_{x} f(x, t) .
$$

The correlations of the line profile still satisfy the dynamic scaling form in Eq.(5), nevertheless with different scaling exponents $\zeta, z$ and scaling function $g$. This self-affine scaling is not critical, i.e., not obtained by fine tuning an external parameter like the force, and is quite different in nature than the critical scaling of the line near the depinning transition, which ceases beyond the correlation length scale $\xi$.

Two important nonperturbative properties of Eq.(40) help us determine these exponents exactly in $1+1$ dimensions:

1. Galilean Invariance (GI): Eq.(40) is statistically invariant under the infinitesimal reparametrization

$$
r^{\prime}=r+\epsilon x, x^{\prime}=x+\lambda \epsilon t, t^{\prime}=t,
$$

provided that the random force $f$ does not have temporal correlations ${ }^{39}$. Since the parameter $\lambda$ appears both in the transformation and Eq.(40), it is not renormalized under any RG procedure that preserves this invariance. This implies the exponent identity ${ }^{38,39}$

$$
\zeta+z=2 .
$$

2. Fluctuation-Dissipation (FD) Theorem: Eqs.(40) and (41) lead to a FokkerPlanck equation for the evolution of the joint probability $\mathcal{P}[r(x)]$,

$$
\partial_{t} \mathcal{P}=\int d x\left(\frac{\delta \mathcal{P}}{\delta r(x)} \partial_{t} r+T \frac{\delta^{2} \mathcal{P}}{[\delta r(x)]^{2}}\right) .
$$

It is easy to check that $\mathcal{P}$ has a stationary solution

$$
\mathcal{P}=\exp \left(-\frac{K}{2 T} \int d x\left(\partial_{x} r\right)^{2}\right)
$$

If $\mathcal{P}$ converges to this solution, the long-time behavior of the correlation functions in Eq.(5) can be directly read off Eq.(46), giving $\zeta=1 / 2$. 
Combining these two results, the roughness and dynamic exponents are exactly determined for the line in two dimensions as

$$
\zeta=1 / 2, \quad z=3 / 2 .
$$

Many direct numerical simulations and discrete growth models have verified these exponents to a very good accuracy. Exact exponents for the isotropic $\mathrm{KPZ}$ equation are not known in higher dimensions, since the FD property is only valid in two dimensions. These results have been summarized in a number of recent reviews ${ }^{40,41,42,43}$.

As an aside we remark that some exact information is available for the anisotropic KPZ equation in $2+1$ dimensions. Using a perturbative RG approach, Wolf showed ${ }^{24}$ that in the equation

$$
\partial_{t} r=K \nabla^{2} r+\frac{\lambda_{x}}{2}\left(\partial_{x} r\right)^{2}+\frac{\lambda_{y}}{2}\left(\partial_{y} r\right)^{2}+f(x, y, t),
$$

the nonlinearities $\left\{\lambda_{x}, \lambda_{y}\right\}$ renormalize to zero if they initially have opposite signs. This suggests logarithmic fluctuations for the resulting interface, as in the case of the linear Langevin equation. In fact, it is straightforward to demonstrate that eq.(48) also satisfies a Fluctuation Dissipation condition if $\lambda_{x}=-\lambda_{y}$. When this condition is satisfied, the associated Fokker-Planck equation has a steady state solution

$$
\mathcal{P}=\exp \left(-\frac{K}{2 T} \int d x d y(\nabla r)^{2}\right) .
$$

This is a non-perturbative result which again indicates the logarithmic fluctuations resulting from eq.(48)In this context, it is interesting to note that the steady state distribution for an exactly solvable discrete model of surface growth belonging to the above universality class has also been obtained ${ }^{44}$

Let us now turn to the case of a line in three dimensions (Fig.5). Fluctuations of the line can be indicated by a a two dimensional vector $\mathbf{r}$. Even in an isotropic medium, the drift velocity $\mathbf{v}$ breaks the isotropy in $\mathbf{r}$ by selecting a direction. A gradient expansion up to second order for the equation of motion gives $^{45}$

$$
\begin{aligned}
\partial_{t} r_{\alpha}=[ & \left.K_{1} \delta_{\alpha \beta}+K_{2} v_{\alpha} v_{\beta}\right] \partial_{x}^{2} r_{\beta} \\
& +\left[\lambda_{1}\left(\delta_{\alpha \beta} v_{\gamma}+\delta_{\alpha \gamma} v_{\beta}\right)+\lambda_{2} v_{\alpha} \delta_{\beta \gamma}+\lambda_{3} v_{\alpha} v_{\beta} v_{\gamma}\right] \frac{\partial_{x} r_{\beta} \partial_{x} r_{\gamma}}{2}+f_{\alpha}
\end{aligned}
$$

with random force correlations

$$
\left\langle f_{\alpha}(x, t) f_{\beta}\left(x^{\prime}, t^{\prime}\right)\right\rangle=2\left[T_{1} \delta_{\alpha \beta}+T_{2} v_{\alpha} v_{\beta}\right] \delta\left(x-x^{\prime}\right) \delta\left(t-t^{\prime}\right) .
$$


Higher order nonlinearities can be similarly constructed but are in fact irrelevant. In terms of components parallel and perpendicular to the velocity, the equations are

$$
\left\{\begin{array}{l}
\partial_{t} r_{\|}=K_{\|} \partial_{x}^{2} r_{\|}+\frac{\lambda_{\|}}{2}\left(\partial_{x} r_{\|}\right)^{2}+\frac{\lambda_{\times}}{2}\left(\partial_{x} r_{\perp}\right)^{2}+f_{\|}(x, t) \\
\partial_{t} r_{\perp}=K_{\perp} \partial_{x}^{2} r_{\perp}+\lambda_{\perp} \partial_{x} r_{\|} \partial_{x} r_{\perp}+f_{\perp}(x, t)
\end{array},\right.
$$

with

$$
\left\{\begin{array}{rl}
\left\langle f_{\|}(x, t) f_{\|}\left(x^{\prime}, t^{\prime}\right)\right\rangle & =2 T_{\|} \delta\left(x-x^{\prime}\right) \delta\left(t-t^{\prime}\right) \\
\left\langle f_{\perp}(x, t) f_{\perp}\left(x^{\prime}, t^{\prime}\right)\right\rangle & =2 T_{\perp} \delta\left(x-x^{\prime}\right) \delta\left(t-t^{\prime}\right)
\end{array} .\right.
$$

The noise-averaged correlations have a dynamic scaling form like Eq.(32),

$$
\left\{\begin{array}{c}
\left\langle\left[r_{\|}(x, t)-r_{\|}\left(x^{\prime}, t^{\prime}\right)\right]^{2}\right\rangle=\left|x-x^{\prime}\right|^{2 \zeta_{\|}} g_{\|}\left(\frac{\left|t-t^{\prime}\right|}{\left|x-x^{\prime}\right|^{z_{\|}}}\right), \\
\left\langle\left[r_{\perp}(x, t)-r_{\perp}\left(x^{\prime}, t^{\prime}\right)\right]^{2}\right\rangle=\left|x-x^{\prime}\right|^{2 \zeta_{\perp}} g_{\perp}\left(\frac{\left|t-t^{\prime}\right|}{\left|x-x^{\prime}\right| z_{\perp}}\right) .
\end{array}\right.
$$

In the absence of nonlinearities $\left(\lambda_{\|}=\lambda_{\times}=\lambda_{\perp}=0\right.$ ), Eqs.(52) can easily be solved to give $\zeta_{\|}=\zeta_{\perp}=1 / 2$ and $z_{\|}=z_{\perp}=2$. Simple dimensional counting indicates that all three nonlinear terms are relevant and may modify the exponents in Eq.(54). Studies of related stochastic equations ${ }^{46,24}$ indicate that interesting dynamic phase diagrams may emerge from the competition between nonlinearities. Let us assume that $\lambda_{\|}$is positive and finite (its sign can be changed by $r_{\|} \rightarrow-r_{\|}$), and focus on the dependence of the scaling exponents on the ratios $\lambda_{\perp} / \lambda_{\|}$and $\lambda_{\times} / \lambda_{\|}$, as depicted in Fig.8. (It is more convenient to set the vertical axis to $\lambda_{\times} K_{\|} T_{\perp} / \lambda_{\|} K_{\perp} T_{\|}$.)

The properties discussed for the KPZ equation can be extended to this higher dimensional case:

1. Galilean Invariance (GI): Consider the infinitesimal reparametrization

$$
\left\{\begin{array}{l}
x^{\prime}=x+\lambda_{\|} \epsilon t, t^{\prime}=t \\
r_{\|}^{\prime}=r_{\|}+\epsilon x, r_{\perp}^{\prime}=r_{\perp} .
\end{array}\right.
$$

Eqs.(52) are invariant under this transformation provided that $\lambda_{\|}=\lambda_{\perp}$. Thus along this line in Fig.8 there is GI, which once more implies the exponent identity

$$
\zeta_{\|}+z_{\|}=2 .
$$




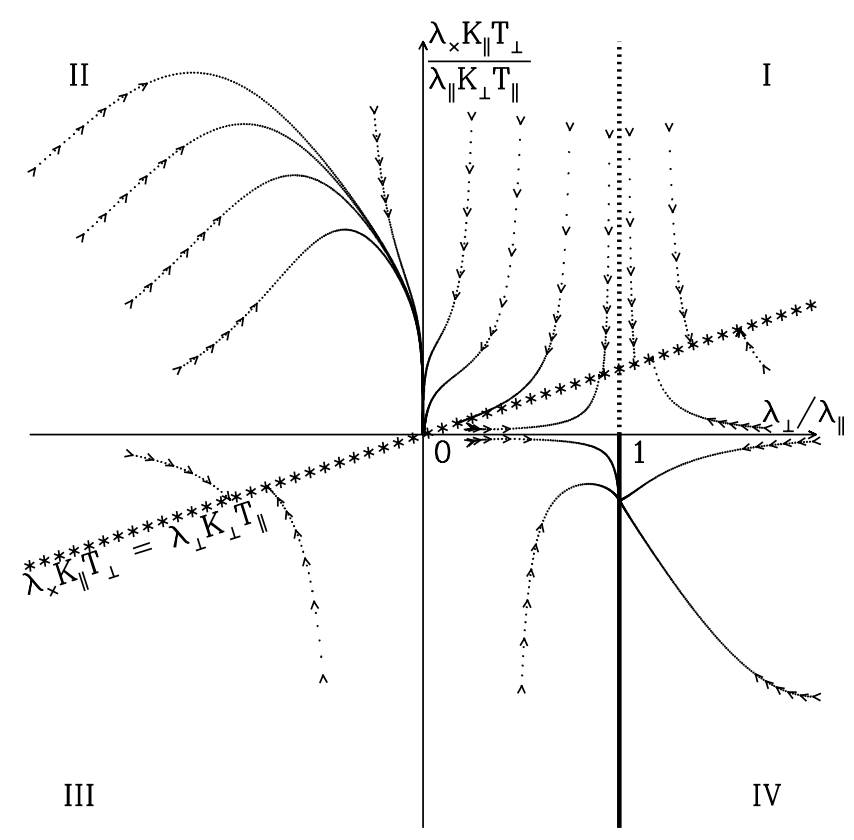

Figure 8. A projection of RG flows in the parameter space, for $n=1$ transverse components.

2. Fluctuation-Dissipation (FD) Condition: The Fokker-Planck equation for the evolution of the joint probability $\mathcal{P}\left[r_{\|}(x), r_{\perp}(x)\right]$ has a stationary solution

$$
\mathcal{P}_{0} \propto \exp \left(-\int d x\left[\frac{K_{\|}}{2 T_{\|}}\left(\partial_{x} r_{\|}\right)^{2}+\frac{K_{\perp}}{2 T_{\perp}}\left(\partial_{x} r_{\perp}\right)^{2}\right]\right)
$$

provided that $\lambda_{\times} K_{\|} T_{\perp}=\lambda_{\perp} K_{\perp} T_{\|}$. Thus for this special choice of parameters, depicted by a starred line in Fig.8, if $\mathcal{P}$ converges to this solution, the longtime behavior of the correlation functions in Eq.(54) can be directly read off Eq.(57), giving $\zeta_{\|}=\zeta_{\perp}=1 / 2$.

3. The Cole-Hopf ( $\mathrm{CH}$ ) Transformation is an important method for the exact study of solutions of the one component nonlinear diffusion equation ${ }^{37}$. Here 
we generalize this transformation to the complex plane by defining, for $\lambda_{\times}<0$,

$$
\Psi(x, t)=\exp \left(\frac{\lambda_{\|} r_{\|}(x, t)+i \sqrt{-\lambda_{\|} \lambda_{\times}} r_{\perp}(x, t)}{2 K}\right) .
$$

The linear diffusion equation

$$
\partial_{t} \Psi=K \partial_{x}^{2} \Psi+\mu(x, t) \Psi
$$

then leads to Eqs.(52) if $K_{\|}=K_{\perp}=K$ and $\lambda_{\|}=\lambda_{\perp}$. [Here $\operatorname{Re}(\mu)=\lambda_{\|} f_{\|} / 2 K$ and $\operatorname{Im}(\mu)=\sqrt{-\lambda_{\|} \lambda_{\times}} f_{\perp} / 2 K$.] This transformation enables an exact solution of the deterministic equation, and further allows us to write the solution to the stochastic equation in the form of a path integral

$$
\Psi(x, t)=\int_{(0,0)}^{(x, t)} \mathcal{D} x(\tau) \exp \left\{-\int_{0}^{t} d \tau\left[\frac{\dot{x}^{2}}{2 K}+\mu(x, \tau)\right]\right\}
$$

Eq.(59) has been extensively studied in connection with quantum tunneling in a disordered medium ${ }^{47}$, with $\Psi$ representing the wave function. In particular, results for the tunneling probability $|\Psi|^{2}$ suggest $z_{\|}=3 / 2$ and $\zeta_{\|}=1 / 2$. The transverse fluctuations correspond to the phase in the quantum problem which is not an observable. Hence this mapping does not provide any information on $\zeta_{\perp}$ and $z_{\perp}$ which are in fact observable for the moving line.

At the point $\lambda_{\perp}=\lambda_{\times}=0, r_{\|}$and $r_{\perp}$ decouple, and $z_{\perp}=2$ while $z_{\|}=3 / 2$. However, in general $z_{\|}=z_{\perp}=z$ unless the effective $\lambda_{\perp}$ is zero. For example at the intersection of the subspaces with GI and FD the exponents $z_{\|}=z_{\perp}=3 / 2$ are obtained from the exponent identities. Dynamic RG recursion relations can be computed to one-loop order ${ }^{45,48}$, by standard methods of momentum-shell dynamic $\mathrm{RG}^{38,39}$.

The renormalization of the seven parameters in Eqs.(52), generalized to 
$n$ transverse directions, give the recursion relations

$$
\begin{aligned}
& \frac{d K_{\|}}{d \ell}=K_{\|}\left[z-2+\frac{1}{\pi} \frac{\lambda_{\|}^{2} T_{\|}}{4 K_{\|}^{3}}+n \frac{1}{\pi} \frac{\lambda_{\perp} \lambda_{\times} T_{\perp}}{4 K_{\|} K_{\perp}^{2}}\right], \\
& \frac{d K_{\perp}}{d \ell}=K_{\perp}\left[z-2+\frac{1}{\pi} \frac{\lambda_{\perp}\left(\left(\lambda_{\times} T_{\perp} / K_{\perp}\right)+\left(\lambda_{\perp} T_{\|} / K_{\|}\right)\right)}{2 K_{\perp}\left(K_{\perp}+K_{\|}\right)}\right. \\
& \left.+\frac{1}{\pi} \frac{K_{\perp}-K_{\|}}{K_{\perp}+K_{\|}} \frac{\left.\left.\lambda_{\perp} T_{\perp} / K_{\perp}\right)-\left(\lambda_{\perp} T_{\|} / K_{\|}\right)\right)}{K_{\perp}\left(K_{\perp}+K_{\|}\right)}\right], \\
& \frac{d T_{\|}}{d \ell}=T_{\|}\left[z-2 \zeta_{\|}-1+\frac{1}{\pi} \frac{\lambda_{\|}^{2} T_{\|}}{4 K_{\|}^{3}}\right]+n \frac{1}{\pi} \frac{\lambda_{\times}^{2} T_{\perp}^{2}}{4 K_{\perp}^{3}}, \\
& \frac{d T_{\perp}}{d \ell}=T_{\perp}\left[z-2 \zeta_{\perp}-1+\frac{1}{\pi} \frac{\lambda_{\perp}^{2} T_{\|}}{K_{\perp} K_{\|}\left(K_{\perp}+K_{\|}\right)}\right], \\
& \frac{d \lambda_{\|}}{d \ell}=\lambda_{\|}\left[\zeta_{\|}+z-2\right], \\
& \frac{d \lambda_{\perp}}{d \ell}=\lambda_{\perp}\left[\zeta_{\|}+z-2-\frac{1}{\pi} \frac{\lambda_{\|}-\lambda_{\perp}}{\left(K_{\perp}+K_{\|}\right)^{2}}\left(\left(\lambda_{\times} T_{\perp} / K_{\perp}\right)-\left(\lambda_{\perp} T_{\|} / K_{\|}\right)\right)\right], \\
& \frac{d \lambda_{\times}}{d \ell}=\lambda_{\times}\left[2 \zeta_{\perp}-\zeta_{\|}+z-2+\frac{1}{\pi} \frac{\lambda_{\|} K_{\perp}-\lambda_{\perp} K_{\|}}{K_{\perp} K_{\|}\left(K_{\perp}+K_{\|}\right)}\left(\left(\lambda_{\times} T_{\perp} / K_{\perp}\right)\right.\right. \\
& \left.\left.-\left(\lambda_{\perp} T_{\|} / K_{\|}\right)\right)\right] .
\end{aligned}
$$

The projections of the RG flows on the two parameter subspace shown in Fig. 8 are indicated by trajectories. They naturally satisfy the constraints imposed by the non-perturbative results: the subspace of GI is closed under RG, while the FD condition appears as a fixed line. The RG flows, and the corresponding exponents, are different in each quadrant of Fig.8, which implies that the scaling behavior is determined by the relative signs of the three nonlinearities. This was confirmed by numerical integrations ${ }^{45,48}$ of Eqs.(52), performed for different sets of parameters. A summary of the computed exponents are given in Table I. follows:

The analysis of analytical and numerical results can be summarized as

$\lambda_{\perp} \lambda_{\times}>0$ : In this region, the scaling behavior is understood best. The RG flows terminate on the fixed line where FD conditions apply, hence $\zeta_{\|}=$ $\zeta_{\perp}=1 / 2$. All along this line, the one loop RG exponent is $z=3 / 2$. These results are consistent with the numerical simulations. The measured exponents rapidly converge to these values, except when $\lambda_{\perp}$ or $\lambda_{\times}$are small.

$\lambda_{\times}=0$ : In this case the equation for $r_{\|}$is the KPZ equation (40), thus $\zeta_{\|}=1 / 2$ and $z_{\|}=3 / 2$. The fluctuations in $r_{\|}$act as a strong (multiplicative and correlated) noise on $r_{\perp}$. The one-loop RG yields the exponents 
$z_{\perp}=3 / 2, \zeta_{\perp}=0.75$ for $\lambda_{\perp}>0$, while a negative $\lambda_{\perp}$ scales to 0 suggesting $z_{\perp}>z_{\|}$. Simulations are consistent with the RG calculations for $\lambda_{\perp}>0$, yielding $\zeta_{\perp}=0.72$, surprisingly close to the one-loop RG value. For $\lambda_{\perp}<0$, simulations indicate $z_{\perp} \approx 2$ and $\zeta_{\perp} \approx 2 / 3$ along with the expected values for the longitudinal exponents.

$\lambda_{\perp}=0$ : The transverse fluctuations satisfy a simple diffusion equation with $\zeta_{\perp}=1 / 2$ and $z_{\perp}=2$. Through the term $\lambda_{\times}\left(\partial_{x} r_{\perp}\right)^{2} / 2$, these fluctuations act as a correlated noise ${ }^{39}$ for the longitudinal mode. A naive application of the results of this reference ${ }^{39}$ give $\zeta_{\|}=2 / 3$ and $z_{\|}=4 / 3$. Quite surprisingly, simulations indicate different behavior depending on the sign of $\lambda_{\times}$. For $\lambda_{\times}<$ $0, z_{\|} \approx 3 / 2$ and $\zeta_{\|} \approx 1 / 2$ whereas for $\lambda_{\times}>0$, longitudinal fluctuations are much stronger, resulting in $z_{\|} \approx 1.18$ and $\zeta_{\|} \approx 0.84$. Actually, $\zeta_{\|}$increases steadily with system size, suggesting a breakdown of dynamic scaling, due to a change of sign in $\lambda_{\perp} \lambda_{\times}$. This dependence on the sign of $\lambda_{\times}$may reflect the fundamental difference between behavior in quadrants II and IV of Fig.8.

TABLE I. Numerical estimates of the scaling exponents, for various values of model parameters for $n=1$. In all cases, $K_{\|}=K_{\perp}=1$ and $T_{\|}=T_{\perp}=0.01$, unless indicated otherwise. Typical error bars are \pm 0.05 for $\zeta, \pm 0.1$ for $z / \zeta$. Entries in brackets are theoretical results. Exact values are given in fractional form.

\begin{tabular}{ccccccc}
\hline \hline$\lambda_{\|}$ & $\lambda_{\times}$ & $\lambda_{\perp}$ & $\zeta_{\|}$ & $z_{\|} / \zeta_{\|}$ & $\zeta_{\perp}$ & $z_{\perp} / \zeta_{\perp}$ \\
\hline 20 & 20 & 20 & 0.48 & 3.0 & 0.48 & 3.0 \\
& & & $(1 / 2)$ & $(3)$ & $(1 / 2)$ & $(3)$ \\
\hline 20 & 20 & 2.5 & 0.75 & 1.7 & 0.50 & 3.7 \\
\hline 20 & 5 & 25 & 0.51 & 3.4 & 0.56 & 2.9 \\
\hline 5 & 5 & -5 & 0.83 & unstable & 0.44 & 3.6 \\
& & & \multicolumn{5}{c}{ (No fixed point for finite $\zeta, z)$} \\
\hline 20 & -20 & -20 & 0.50 & 3.1 & 0.50 & 2.9 \\
& & & $(1 / 2)$ & $(3)$ & $(1 / 2)$ & $(3)$ \\
\hline 5 & -5 & 5 & 0.52 & 3.3 & 0.57 & 3.4 \\
& & & $(1 / 2)$ & $(3)$ & $($ Strong coupling $)$ \\
\hline 20 & 0 & 20 & 0.49 & 3.1 & 0.72 & 2.2 \\
& & & $(1 / 2)$ & $(3)$ & $(0.75)$ & $(2)$ \\
\hline 20 & 0 & -20 & 0.48 & 3.0 & 0.65 & 3.1 \\
& & & $(1 / 2)$ & $(3)$ & & $\left(z_{\perp}>z_{\|}\right)$ \\
\hline 20 & 20 & 0 & 0.84 & 1.4 & 0.50 & 4.0 \\
& & & \multicolumn{2}{c}{$\left(z_{\|}<z_{\perp}\right)$} & $(1 / 2)$ & $(4)$ \\
\hline 20 & -20 & 0 & 0.55 & 2.9 & 0.51 & 4.0 \\
& & & $\left(z_{\|}<z_{\perp}\right)$ & $(1 / 2)$ & $(4)$ \\
\hline \hline
\end{tabular}

$\lambda_{\perp}<0$ and $\lambda_{\times}>0$ : The analysis of this region (II) is the most difficult in that the RG flows do not converge upon a finite fixed point and $\lambda_{\perp} \rightarrow 0$, which 
may signal the breakdown of dynamic scaling. Simulations indicate strong longitudinal fluctuations that lead to instabilities in the discrete integration scheme, excluding the possibility of measuring the exponents reliably.

$\lambda_{\perp}>0$ and $\lambda_{\times}<0$ : The projected RG flows in this quadrant (IV) converge to the point $\lambda_{\perp} / \lambda_{\|}=1$ and $\lambda_{\times} T_{\perp} K_{\|} / \lambda_{\|} T_{\|} K_{\perp}=-1$. This is actually not a fixed point, as $K_{\|}$and $K_{\perp}$ scale to infinity. The applicability of the $\mathrm{CH}$ transformation to this point implies $z_{\|}=3 / 2$ and $\zeta_{\|}=1 / 2$. Since $\lambda_{\perp}$ is finite, $z_{\perp}=z_{\|}=3 / 2$ is expected, but this does not give any information on $\zeta_{\perp}$. Simulations indicate strong transverse fluctuations and suffer from difficulties similar to those in region II.

Eqs. (52) are the simplest nonlinear, local, and dissipative equations that govern the fluctuations of a moving line in a random medium. They can be easily generalized to describe the time evolution of a manifold with arbitrary internal $\left(\mathrm{x} \in R^{d}\right)$ and external $\left(\mathbf{r} \in R^{n+1}\right)$ dimensions, and to the motion of curves that are not necessarily stretched in a particular direction. Since the derivation only involves general symmetry arguments, the given results are widely applicable to a number of seemingly unrelated systems. We will discuss one application to drifting polymers in more detail in the next lecture, explicitly demonstrating the origin of the nonlinear terms starting from more fundamental hydrodynamic equations. A simple model of crack front propagation in three dimensions ${ }^{49}$ also arrives at Eqs.(52), implying the self-affine structure of the crack surface after the front has passed.

\subsection{Drifting Polymers}

The dynamics of polymers in fluids is of much theoretical interest and has been extensively studied ${ }^{50,51}$. The combination of polymer flexibility, interactions, and hydrodynamics make a first principles approach to the problem quite difficult. There are, however, a number of phenomenological studies that describe various aspects of this problem ${ }^{52}$.

One of the simplest is the Rouse model ${ }^{53}$ : The configuration of the polymer at time $t$ is described by a vector $\mathbf{R}(x, t)$, where $x \in[0, N]$ is a continuous variable replacing the discrete monomer index (see Fig.9).

Ignoring inertial effects, the relaxation of the polymer in a viscous medium is approximated by

$$
\partial_{t} \mathbf{R}(x, t)=\mu \mathbf{F}(\mathbf{R}(x, t))=K \partial_{x}^{2} \mathbf{R}(x, t)+\eta(x, t),
$$

where $\mu$ is the mobility. The force $\mathbf{F}$ has a contribution from interactions with near neighbors that are treated as springs. Steric and other interactions are ignored. The effect of the medium is represented by the random forces $\eta$ with zero mean. The Rouse model is a linear Langevin equation that is easily solved. It predicts that the mean square radius of gyration, $R_{g}^{2}=\left\langle|\mathbf{R}-\langle\mathbf{R}\rangle|^{2}\right\rangle$, is proportional to the polymer size $N$, and the largest relaxation times scale 


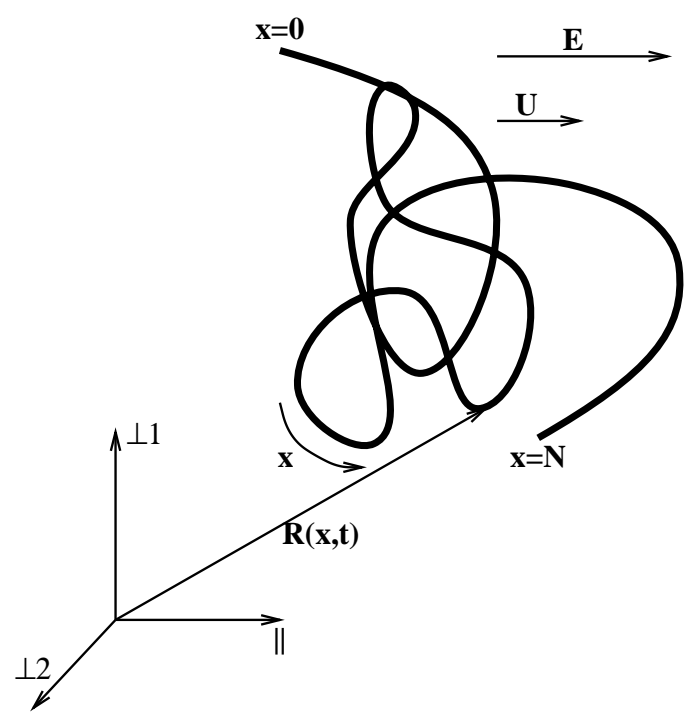

Figure 9. The configuration of a polymer.

as the fourth power of the wave number, (i.e., in dynamic light scattering experiments, the half width at half maximum of the scattering amplitude scales as the fourth power of the scattering wave vector $\mathbf{q})$. These results can be summarized as $R_{g} \sim N^{\nu}$ and $\Gamma(\mathbf{q}) \sim q^{z}$, where $\nu$ and $z$ are called the swelling and dynamic exponents, respectively ${ }^{54}$. Thus, for the Rouse Model, $\nu=1 / 2$ and $z=4$.

The Rouse model ignores hydrodynamic interactions mediated by the fluid. These effects were originally considered by Kirkwood and Risemann ${ }^{55}$ and later on by Zimm ${ }^{56}$. The basic idea is that the motion of each monomer modifies the flow field at large distances. Consequently, each monomer experiences an additional velocity

$\delta_{H} \partial_{t} \mathbf{R}(x, t)=\frac{1}{8 \pi \eta_{s}} \int d x^{\prime} \frac{\mathbf{F}\left(x^{\prime}\right) r_{x x^{\prime}}^{2}+\left(\mathbf{F}\left(x^{\prime}\right) \cdot \mathbf{r}_{x x^{\prime}}\right) \mathbf{r}_{x x^{\prime}}}{\left|\mathbf{r}_{x x^{\prime}}\right|^{3}} \approx \int d x^{\prime} \frac{\gamma}{\left|x-x^{\prime}\right|^{\nu}} \partial_{x}^{2} \mathbf{R}$,

where $\mathbf{r}_{x x^{\prime}}=\mathbf{R}(x)-\mathbf{R}\left(x^{\prime}\right)$ and the final approximation is obtained by replacing the actual distance between two monomers by their average value. The modified equation is still linear in $\mathbf{R}$ and easily solved. The main result is the speeding up of the relaxation dynamics as the exponent $z$ changes from 4 to 3. Most experiments on polymer dynamics ${ }^{57}$ indeed measure exponents close to 3. Rouse dynamics is still important in other circumstances, such as diffusion of a polymer in a solid matrix, stress and viscoelasticity in concentrated 
polymer solutions, and is also applicable to relaxation times in Monte Carlo simulations.

Since both of these models are linear, the dynamics remains invariant in the center of mass coordinates upon the application of a uniform external force. Hence the results for a drifting polymer are identical to a stationary one. This conclusion is in fact not correct due to the hydrodynamic interactions. For example, consider a rodlike conformation of the polymer with monomer length $b_{0}$ where $\partial_{x} R_{\alpha}=b_{0} t_{\alpha}$ everywhere on the polymer, so that the elastic (Rouse) force vanishes. If a uniform force $\mathbf{E}$ per monomer acts on this rod, the velocity of the rod can be solved using Kirkwood Theory, and the result is ${ }^{50}$

$$
\mathbf{v}=\frac{(-\ln \kappa)}{4 \pi \eta_{s} b_{0}} \mathbf{E} \cdot[\mathbf{I}+\mathbf{t t}]
$$

In the above equation, $\eta_{s}$ is the solvent viscosity, $\mathbf{t}$ is the unit tangent vector, $\kappa=2 b / b_{0} N$ is the ratio of the width $b$ to the half length $b_{0} N / 2$ of the polymer. A more detailed calculation of the velocity in the more general case of an arbitrarily shaped slender body by Khayat and $\mathrm{Cox}^{58}$ shows that nonlocal contributions to the hydrodynamic force, which depend on the whole shape of the polymer rather than the local orientation, are $\mathcal{O}\left(1 /(\ln \kappa)^{2}\right)$. Therefore, corrections to Eq.(63) are small when $N \gg b / b_{0}$.

Incorporating this tilt dependence of polymer mobility requires adding terms nonlinear in the tilt, $\partial_{x} \mathbf{r}$, to a local equation of motion. Since the overall force (or velocity) is the only vector breaking the isotropy of the fluid, the structure of these nonlinear terms must be identical to eq.(50). Thus in terms of the fluctuations parallel and perpendicular to the average drift, we again recover the equations,

$$
\left\{\begin{array}{c}
\partial_{t} R_{\|}=U_{\|}+K_{\|} \partial_{x}^{2} R_{\|}+\frac{\lambda_{\|}}{2}\left(\partial_{x} R_{\|}\right)^{2}+\frac{\lambda_{\times}}{2} \sum_{i=1}^{2}\left(\partial_{x} R_{\perp i}\right)^{2}+\eta_{\|}(x, t), \\
\partial_{t} R_{\perp i}=K_{\perp} \partial_{x}^{2} R_{\perp i}+\lambda_{\perp} \partial_{x} R_{\|} \partial_{x} R_{\perp i}+\eta_{\perp i}(x, t),
\end{array}\right.
$$

where $\{\perp i\}$ refers to the 2 transverse coordinates of the monomer positions. The noise is assumed to be white and gaussian but need not be isotropic, i.e.

$$
\left\{\begin{aligned}
\left\langle\eta_{\|}(x, t) \eta_{\|}\left(x^{\prime}, t^{\prime}\right)\right\rangle & =2 T_{\|} \delta\left(x-x^{\prime}\right) \delta\left(t-t^{\prime}\right), \\
\left\langle\eta_{\perp i}(x, t) \eta_{\perp j}\left(x^{\prime}, t^{\prime}\right)\right\rangle & =2 T_{\perp} \delta_{i, j} \delta\left(x-x^{\prime}\right) \delta\left(t-t^{\prime}\right) .
\end{aligned}\right.
$$

At zero average velocity, the system becomes isotropic and the equations of motion must coincide with the Rouse model. Therefore, $\left\{\lambda_{\|}, \lambda_{\times}, \lambda_{\perp}, U, K_{\|}-\right.$ 
$\left.K_{\perp}, T_{\|}-T_{\perp}\right\}$ are all proportional to $E$ for small forces. The relevance of these nonlinear terms are determined by the dimensionless scaling variable

$$
y=\left(\frac{U}{U^{*}}\right) N^{1 / 2},
$$

where $U^{*}$ is a characteristic microscopic velocity associated with monomer motion and is roughly $10-20 \mathrm{~m} / \mathrm{s}$ for polystyrene in benzene. The variable $y$ is proportional to another dimensionless parameter, the Reynolds number $R e$, which determines the breakdown of hydrodynamic equations and onset of turbulence. However, typically $R e \ll y$, and the hydrodynamic equations are valid for moderately large $y$. Eqs. (64) describe the static and dynamical scaling properties of the nonlinear and anisotropic regime when $U>U^{*} N^{-1 / 2}$.

Eq.(64) is just a slight variation from (52), with two transverse components instead of one. Thus, the results discussed in the previous lecture apply. A more detailed calculation of the nonlinear terms from hydrodynamics ${ }^{59}$ shows that all three nonlinearities are positive for small driving forces. In this case, the asymptotic scaling exponents are isotropic, with $\nu=1 / 2$ and $z=3$. However, the fixed points of the RG transformation are in general anisotropic, which implies a kinetically induced form birefringence in the absence of external velocity gradients. This is in marked contrast with standard theories of polymer dynamics where a uniform driving force has essentially no effect on the internal modes of the polymer.

When one of the nonlinearities approaches to zero, the swelling exponents may become anisotropic and the polymer elongates or compresses along the longitudinal direction. However, the experimental path in the parameter space as a function of $E$ is not known and not all of the different scaling regimes correspond to actual physical situations. The scaling results found by the RG analysis are verified by direct integration of equations, as mentioned in the earlier lectures. A more detailed discussion of the analysis and results can be found in our earlier work ${ }^{48}$.

In constructing equations (64), we only allowed for local effects, and ignored the nonlocalities that are the hallmark of hydrodynamics. One consequence of hydrodynamic interactions is the back-flow velocity in Eq.(62) that can be added to the evolution equations (64). Dimensional analysis gives the recursion relation

$$
\frac{\partial \gamma}{\partial \ell}=\gamma[\nu z-1-(d-2) \nu]+O\left(\gamma^{2}\right),
$$

which implies that, at the nonlinear fixed point, this additional term is surprisingly irrelevant for $d>3$, and $z=3$ due to the nonlinearities. For $d<3$, $z=d$ due to hydrodynamics, and the nonlinear terms are irrelevant. The situation in three dimensions is unclear, but a change in the exponents is unlikely. 
Similarly, one could consider the effect of self-avoidance by including the force generated by a softly repulsive contact potential

$$
\frac{b}{2} \int d x d x^{\prime} \mathcal{V}\left(\mathbf{r}(x)-\mathbf{r}\left(x^{\prime}\right)\right) .
$$

The relevance of this term is also controlled by the scaling dimension $y_{b}=$ $\nu z-1-(d-2) \nu$, and therefore this effect is marginal in three dimensions at the nonlinear fixed point, in contrast with both Rouse and Zimm models where self-avoidance becomes relevant below four dimensions. Unfortunately, one is ultimately forced to consider non-local and nonlinear terms based on similar grounds, and such terms are indeed relevant below four dimensions. In some cases, local or global arclength conservation may be an important consideration in writing down a dynamics for the system. However, a local description is likely to be more correct in a more complicated system with screening effects (motion in a gel that screens hydrodynamic interactions) where a first principles approach becomes even more intractable. Therefore, this model is an important starting point towards understanding the scaling behavior of polymers under a uniform drift, a problem with great technological importance.

\section{Acknowledgments}

The work described here is part of the doctoral thesis of Deniz Ertas in the Physics Department of MIT. Financial support from the NSF through grant number DMR-93-03667 is gratefully acknowledged. Many thanks are due to Drs. D. Kim, Y. Kim, J.M. Kim, I.-M. Kim, H. Park, and B. Kahng for organizing the 4th CTP Workshop on Statistical Physics, and providing the opportunity for these lectures.

\section{REFERENCES}

1. H. Fukuyama and P. A. Lee, Phys. Rev. B 17, 535 (1978); P. A. Lee and T. M. Rice, Phys. Rev. B 19, 3970 (1979).

2. R. Bruinsma and G. Aeppli, Phys. Rev. Lett. 52, 1547 (1984); J. Koplik and H. Levine, Phys. Rev. B 32, 280 (1985).

3. P.G. de Gennes, Rev. Mod. Phys. 57, 827 (1985).

4. J.F. Joanny and P.G. de Gennes, J. Chem. Phys. 81, 552 (1984).

5. D.S. Fisher, Phys. Rev. Lett. 50, 1486 (1983).

6. D.S. Fisher, Phys. Rev. B 31, 1396 (1985), O. Narayan and D.S. Fisher, Phys. Rev. B 46, 11520 (1992).

7. T. Nattermann, S. Stepanow, L.-H. Tang, and H. Leschhorn, J. Phys. II France 2, 1483 (1992).

8. Y. Imry and S.-K. Ma, Phys. Rev. Lett. 35, 1399 (1975). 
9. P.C. Martin, E. Siggia, and H. Rose, Phys. Rev. A 8, 423 (1973).

10. O. Narayan and D. S. Fisher, Phys. Rev. B 48, 7030 (1993).

11. M. Dong, M. C. Marchetti, A. A. Middleton, and V. Vinokur, Phys. Rev. Lett. 70, 662 (1993). The identification of the exponent $\zeta=1$ from correlation function has been questioned by H. Leschhorn and L.-H. Tang, Phys. Rev. Lett. 70, 2973 (1993).

12. M.A. Rubio, C.A. Edwards, A. Dougherty, and J.P Gollub, Phys. Rev. Lett. 63, 1685 (1989); V.K. Horváth, F. Family, and T. Vicsek, Phys. Rev. Lett. 67, 3207 (1991); S. He, G. L. M. K. S. Kahanda, and P.-Z. Wong, Phys. Rev. Lett. 69, 3731 (1992).

13. S. V. Buldyrev, A.-L. Barabasi, F. Caserta, S. Havlin, H. E. Stanley, and T. Vicsek, Phys. Rev. A 45, R8313 (1992).

14. F. Family, K. C. B. Chan, and J. G. Amar, in Surface Disordering: Growth, Roughening and Phase Transitions, Les Houches Series, Nova Science Publishers, New York (1992).

15. H. Leschhorn, Physica A 195, 324 (1993).

16. L. A. N. Amaral, A.-L. Barabasi, and H. E. Stanley, Phys. Rev. Lett. 73, 62 (1994).

17. H. Ji and M. O. Robbins, Phys. Rev. B 44, 2538 (1991); B. Koiller, H. Ji, and M. O. Robbins, Phys. Rev. B 46, 5258 (1992).

18. L.-H. Tang and H. Leschhorn, Phys. Rev. A 45, R8309 (1992).

19. M. Kardar, G. Parisi, and Y.-C. Zhang, Phys. Rev. Lett. 56, 889 (1986).

20. C. Tang, S. Feng, and L. Golubovic, Phys. Rev. Lett. 72, 1264 (1994).

21. S. Stepanow, J. Phys. II France 5, 11 (1995).

22. Z. Chahók, K. Honda, and T. Vicsek, J. Phys. A 26, L171 (1993); S. Galluccio and Y.-C. Zhang, Phys. Rev. E 51, 1686 (1995); H. Leschhorn, cond-mat 9605018.

23. D. Dhar, M. Barma, and M.K. Phani, Phys. Rev. Lett. 47, 1238 (1981); D. Dhar, J. Phys. A 15, 1859 (1982); S.V. Buldyrev, S. Havlin, and H.E. Stanley, Physica 200, 200 (1993); and references therein.

24. D. Wolf, Phys. Rev. Lett. 67, 1783 (1991).

25. H. Jeong, B. Kahng, and D. Kim, Phys. Rev. Lett. 77, 5094 (1996).

26. See, for example, G. Blatter, M. V. Feigel'man, V. B. Geshkenbein, A. I. Larkin, and V. M. Vinokur, Rev. Mod. Phys. 66, 1125 (1994); and references therein.

27. R. H. Koch et al., Phys. Rev. Lett. 63, 1511 (1989); P. L. Gammel, L. F. Schneemener, and D.J. Bishop, Phys. Rev. Lett. 66, 953 (1991).

28. L. Civale et al., Phys. Rev. Lett. 67, 648 (1991); M. Leghissa et al., Phys. Rev. B 48, 1341 (1993).

29. D. S. Fisher, M. P. A. Fisher, and D. A. Huse, Phys. Rev. B 43, 130 (1991).

30. D. R. Nelson and V. M. Vinokur, Phys. Rev. Lett. 68, 2398 (1992). 
31. Y. Enomoto, Phys. Lett. A 161, 185 (1991); Y. Enomoto, K. Katsumi, R. Kato, and S. Maekawa, Physica C 192, 166 (1992).

32. A. A. Middleton and D. S. Fisher, Phys. Rev. Lett. 66, 92 (1991); Phys. Rev. B 47, 3530 (1993).

33. H. Sompolinsky and A. Zippelius, Phys. Rev. B 25, 6860 (1982); A. Zippelius, Phys. Rev. B 29, 2717 (1984).

34. In this case, the longitudinal direction is chosen to be along the average velocity $\mathbf{v}$, not the Lorentz force $\mathbf{F}$.

35. M. Kardar, in Disorder and Fracture, edited by J.C. Charmet, S. Roux, and E. Guyon, Plenum, New York (1990); T. Hwa and M. Kardar, Phys. Rev. A 45, 7002 (1992).

36. M. Plischke, Z. Rácz, and D. Liu, Phys. Rev. B 35, 3485 (1987).

37. J.M. Burgers, The Nonlinear Diffusion Equation (Riedel, Boston, 1974).

38. D. Forster, D. R. Nelson, and M. J. Stephen, Phys. Rev. A 16, 732 (1977).

39. E. Medina, T. Hwa, M. Kardar, and Y. Zhang, Phys. Rev. A 39, 3053 (1989).

40. Dynamics of Fractal Surfaces, edited by F. Family and T. Vicsek (World Scientific, Singapore, 1991).

41. J. Krug and H. Spohn, in Solids Far From Equilibrium: Growth, Morphology and Defects, edited by C. Godreche (Cambridge University Press, Cambridge, 1991).

42. T. Halpin-Healy and Y.-C. Zhang, Phys. Rep. 254, 215 (1995).

43. A.-L. Barabasi and H. E. Stanley, Fractal concepts in surface growth, (CUP, Cambridge, 1995).

44. M. Prahofer and H. Spohn, J. Stat. Phys., in press (1997).

45. D. Ertas and M. Kardar, Phys. Rev. Lett. 69, 929 (1992).

46. T. Hwa, Phys. Rev. Lett. 69, 1552 (1992).

47. E. Medina, M. Kardar, Y. Shapir, and X.-R. Wang, Phys. Rev. Lett. 62, 941 (1989); E. Medina and M. Kardar, Phys. Rev. B 46, 9984 (1992).

48. D. Ertas and M. Kardar, Phys. Rev. E 48, 1228 (1993).

49. J. P. Bouchaud, E. Bouchaud, G. Lapasset, and J. Planes, Phys. Rev. Lett. 71, 2240 (1993).

50. M. Doi and S.F. Edwards, Theory of Polymer Dynamics, Oxford University Press (1986).

51. P.G. de Gennes, Scaling Concepts in Polymer Physics, Cornell University Press (1979).

52. R.B. Bird, Dynamics of Polymeric Physics, Vols. 1-2, Wiley, New York (1987).

53. P.E. Rouse, J. Chem. Phys. 21, 1272 (1953).

54. We have changed the notation to confer with the traditions of polymer science. $\nu$ is $\zeta$ and $z$ is $z / \zeta$ in terms of the notation used previously. 
55. J. Kirkwood and J. Risemann, J. Chem. Phys. 16, 565 (1948).

56. B.H. Zimm, J. Chem. Phys. 24, 269 (1956).

57. See, for example, M. Adam and M. Delsanti, Macromolecules 10, 1229 (1977).

58. R.E. Khayat and R.G. Cox, J. Fluid. Mech. 209, 435 (1989).

59. See Appendices A and B of our longer paper ${ }^{48}$. 